\title{
COORDINATION AND GEOMETRIC OPTIMIZATION VIA DISTRIBUTED DYNAMICAL SYSTEMS*
}

\author{
JORGE CORTÉS ${ }^{\dagger}$ AND FRANCESCO BULLO ${ }^{\dagger}$
}

\begin{abstract}
This paper discusses dynamical systems for disk-covering and sphere-packing problems. We present facility location functions from geometric optimization and characterize their differentiable properties. We design and analyze a collection of distributed control laws that are related to nonsmooth gradient systems. The resulting dynamical systems promise to be of use in coordination problems for networked robots; in this setting the distributed control laws correspond to local interactions between the robots. The technical approach relies on concepts from computational geometry, nonsmooth analysis, and the dynamical system approach to algorithms.
\end{abstract}

Key words. distributed dynamical systems, coordination and cooperative control, geometric optimization, disk-covering problem, sphere-packing problem, nonsmooth analysis, Voronoi partitions.

AMS subject classifications. 37N35, 68W15, 93D20, 49J52, 05B40

1. Introduction. Consider $n$ sites $\left(p_{1}, \ldots, p_{n}\right)$ evolving within a convex polygon $Q$ according to one of the following interaction laws: (i) each site moves away from the closest other site or polygon boundary, (ii) each site moves toward the furthest vertex of its own Voronoi polygon, or (iii) each site moves toward a geometric center (circumcenter, incenter, centroid, etc) of its own Voronoi polygon. Recall that the Voronoi polygon of the $i$ th site is the closed set of points $q \in Q$ closer to $p_{i}$ than to any other $p_{j}$.

These and related interaction laws give rise to strikingly simple dynamical systems whose behavior remains largely unknown. What are the critical points of such dynamical systems? What is their asymptotic behavior? Are these systems optimizing any aggregate function? In what way do these local interactions give rise to distributed systems? Does any biological ensemble evolve according to these behaviors and are they of any engineering use in coordination problems? These are the questions that motivate this paper.

Coordination in robotics, control, and biology. Coordination problems are becoming increasingly important in numerous engineering disciplines. The deployment of large groups of autonomous vehicles is rapidly becoming possible because of technological advances in computing, networking, and miniaturization of electromechanical systems. These future multi-vehicle networks will coordinate their actions to perform challenging spatially-distributed tasks (e.g., search and recovery operations, exploration, surveillance, and environmental monitoring for pollution detection and estimation). This future scenario motivates the study of algorithms for autonomy, adaptation, and coordination of multi-vehicle networks. It is also important to take into careful consideration all constraints on the behavior of the multi-vehicle network. Coordination algorithms need to be adaptive and distributed in order for the resulting closed-loop network to be scalable, to comply with bandwidth limitations, to tolerate

\footnotetext{
*Submitted to the SIAM Journal on Control and Optimization on May 27, 2003. This work was supported by DARPA/AFOSR MURI Award F49620-02-1-0325. A preliminary version of this manuscript has been submitted to the 2003 IEEE Control and Decision Conference, Maui, Hawaii.

${ }^{\dagger}$ Coordinated Science Laboratory, University of Illinois at Urbana-Champaign, 1308 W. Main St., Urbana, IL 61801, United States, Ph. +1 217 244-8734 and +1 217 333-0656, Fax. +1 217 244-1653, \{jcortes, bullo\}@uiuc.edu, http://motion.csl.uiuc.edu/ $\{$ jorge, bullo\}
} 
failures, and to adapt to changing environments, topologies and sensing tasks. The interaction laws introduced above have these properties and, remarkably, they optimize network-wide performance measures for meaningful spatially-distributed tasks.

Coordinated group motions are also a widespread phenomenon in biological systems. Some species of fish spend their lives in schools as a defense mechanism against predators. Others travel as swarms in order to protect an area that they have claimed as their own. Flocks of birds are able to travel in large groups and act as one unit. Other animals exhibit remarkable collective behaviors when foraging and selecting food. Certain foraging behaviors include individual animals partitioning their environment in nonoverlapping individual zones whereas other species develop overlapping team areas. These biological network systems possess extraordinary dynamic capabilities without apparently following a group leader. Yet, these complex coordinated behaviors emerge while each individual has no global knowledge of the network state and can only plan its motion according to the observation of its closest neighbors.

Facility location, nonsmooth stability analysis and cooperative control. To analyze the interaction laws introduced above we rely on concepts and methods from various disciplines. Facility location problems play a prominent role in the field of geometric optimization [1, 5]. Facility location pervades a broad spectrum of scientific and technological areas, including resource allocation (where to place mailboxes in a city or cache servers on the internet), quantization and information theory, mesh and grid optimization methods, clustering analysis, data compression, and statistical pattern recognition. Smooth multi-center functions for so-called centroidal Voronoi configurations and smooth distributed dynamical systems are presented in [10, 14. Multi-center functions are studied in resource allocation problems [13, 29] and in quantization theory [16 21]. The role of Voronoi tessellations and computational geometry in facility location is discussed in 23, 26].

The notion and computational properties of the generalized gradient are throughly studied in nonsmooth analysis 9. In particular, tools for establishing stability and convergence properties of nonsmooth dynamical systems are presented in 3, 15, 27. Finally, we refer to [7, 17] for guidelines on how to design dynamical systems for optimization purposes, and to 4 for gradient descent flows in distributed computation in settings with fixed-communication topologies.

Recent years have witnessed a large research effort focused on motion planning and formation control problems for multi-vehicle systems [12, 18, 19, 20, 24, 30, 31]. Within the literature on behavior-based robotics, heuristic approaches to the design of interaction rules and emerging behaviors have been investigated (see 22 and references therein). Along this specific line of research, no formal results guaranteeing the correctness of the proposed algorithms or their optimality with respect to an aggregate objective are currently available. The aim of this work is to design distributed coordination algorithms for dynamic networks as well as to provide formal verifications of their asymptotic correctness. A key aspect of our treatment is the inherent complexity of studying networks whose communication topology changes along the system evolution, as opposed to networks with fixed communication topologies. This key aspect is present in the analysis of distributed control laws in [18, 30, 31] and of agreement protocols in [24].

Statement of contributions. We consider two facility location functions from geometric optimization that characterize coverage performance criteria. A collection of sites provides optimal service to a domain of interest if (i) it minimizes the largest distance from any point in the domain to one of the sites, or (ii) it maximizes the 
minimum distance between any two sites. In other words, if $P=\left(p_{1}, \ldots, p_{n}\right)$ are $n$ sites evolving within a convex polygon $Q$, we extremize the multi-center functions

$$
\max _{q \in Q}\left\{\min _{i \in\{1, \ldots, n\}} d\left(q, p_{i}\right)\right\}, \quad \min _{i \neq j \in\{1, \ldots, n\}}\left\{\frac{1}{2} d\left(p_{i}, p_{j}\right), d\left(p_{i}, \partial Q\right)\right\},
$$

where $d(p, q)$ and $d(p, \partial Q)$ are the distances between $p$ and $q$, and between $p$ and the boundary of $Q$, respectively. (The role of the $\frac{1}{2}$ factor will become clear later.) We study the differentiable properties of these functions via nonsmooth analysis. We show the functions are globally Lipschitz and regular, we compute their generalized gradients, and we characterize their critical points. Under certain technical conditions, we show that the local minima of the first multi-center function are so-called circumcenter Voronoi configurations, and that these critical points correspond to the solutions of disk-covering problems. Similarly, under analogous technical conditions, we show that the local maxima of the second multi-center function are so-called incenter Voronoi configurations, and that these critical points correspond to the solutions of sphere-packing problems.

Next, we aim to design distributed algorithms that extremize the multi-center functions. Roughly speaking, by distributed we mean that the evolution of each site depends at most on the location of its own Voronoi neighbors. We study the generalized gradient flows induced by the multi-center functions using nonsmooth stability analysis. Although these dynamical systems possess some convergence properties, they are not amenable to distributed implementations. Next, drawing connections with quantization theory, we consider two dynamical systems associated to each multicenter function. First, we consider a novel strategy based on the generalized gradient of the 1-center functions of each site, and, second, we consider a geometric centering strategy similar to the well-known Lloyd algorithm [16, 21.

Remarkably, these strategies arising from the nonsmooth gradient information have natural geometric interpretations and are indeed the local interaction rule described earlier. For the first (respectively second) multi-center function, the first strategy corresponds to the interaction law "move toward the furthest vertex of own Voronoi polygon" (respectively, "move away from the closest other site or polygon boundary", and the second strategy corresponds to the interaction law "move toward circumcenter of own Voronoi polygon" (respectively "move toward incenter of own Voronoi polygon"). We prove the uniqueness of the solutions of the resulting distributed dynamical systems and we analyze their asymptotic behavior using nonsmooth stability analysis, showing that the active sites will approach the corresponding centers of their own Voronoi cells.

Two of our results are related to well-known conjectures in the locational optimization literature [13, 29]: (i) that the first multi-center problem is equivalent to a disk covering problem (how to cover a region with possibly overlapping disks of equal minimum radius), and (ii) that the generalized Lloyd strategy "move toward circumcenter of own Voronoi polygon" converges to the set of circumcenter Voronoi configurations.

Organization. The paper is organized as follows. Section 2 provides the preliminary concepts on Voronoi partitions, nonsmooth analysis, stability analysis, and gradient flows, and introduces the multi-center problems. Section 3 presents a complete treatment on the functions analysis and algorithm design for the 1-center problems. Section 4 discusses the differentiable properties and the critical points of the multi-center functions. Section 5 introduces a number of dynamical systems (smooth 
and nonsmooth, distributed and non-distributed) and analyzes their asymptotic correctness.

2. Preliminaries and problem setup. Let $\|\cdot\|$ denote the Euclidean distance function on $\mathbb{R}^{N}$ and let $v \cdot w$ denote the scalar product of the vectors $v, w \in \mathbb{R}^{N}$. Let $\operatorname{vrs}(v)$ denote the unit vector in the direction of $0 \neq v \in \mathbb{R}^{N}$, i.e., $\operatorname{vrs}(v)=v /\|v\|$. Given a set $S$ in $\mathbb{R}^{N}$, we denote its convex hull by $\operatorname{co}(S)$ and its interior set by $\operatorname{int}(S)$. If $S$ is a convex set in $\mathbb{R}^{N}$, let $\operatorname{proj}_{S}: \mathbb{R}^{N} \rightarrow S$ denote the orthogonal projection onto $S$ and let $\mathrm{D}_{S}: \mathbb{R}^{N} \rightarrow \mathbb{R}$ denote the distance function to $S$. For $R>0$, let $\bar{B}_{N}(p, R)=\left\{q \in \mathbb{R}^{N} \mid\|p-q\| \leq R\right\}$, and $B_{N}(p, R)=\operatorname{int}\left(\bar{B}_{N}(p, R)\right)$. A set $\left\{v_{1}, \ldots, v_{M}\right\}$ of vectors in $\mathbb{R}^{N}$ positively spans $\mathbb{R}^{N}$ if any $w \in \mathbb{R}^{N}$ can be written as $w=\sum_{l=1}^{M} a_{l} v_{l}$, with $a_{l} \geq 0, l \in\{1, \ldots, M\}$. The following simple lemma, e.g., see [8], characterizes this situation.

Lemma 2.1. Given a set $\left\{v_{1}, \ldots, v_{M}\right\}$ of $M$ arbitrary vectors in $\mathbb{R}^{N}$, then the following statements are equivalent

(i) $\left\{v_{1}, \ldots, v_{M}\right\}$ positively spans $\mathbb{R}^{N}$;

(ii) $0 \in \operatorname{int}\left(\operatorname{co}\left\{v_{1}, \ldots, v_{M}\right\}\right)$;

(iii) for each $w \in \mathbb{R}^{N}$, there exists $v_{i}$ such that $w \cdot v_{i}>0$.

Let $Q$ be a convex polygon in $\mathbb{R}^{2}$. We denote by $\operatorname{Ed}(Q)=\left\{e_{1}, \ldots, e_{M}\right\}$ and $\operatorname{Ve}(Q)=\left\{v_{1}, \ldots, v_{L}\right\}$ the set of edges and vertexes of $Q$, respectively. Let $P=$ $\left(p_{1}, \ldots, p_{n}\right) \in Q^{n} \subset\left(\mathbb{R}^{2}\right)^{n}$ denote the location of $n$ generators in the space $Q$. Let $\pi_{i}: Q^{n} \rightarrow Q$ be the canonical projection onto the $i$ th factor, $\pi_{i}\left(p_{1}, \ldots, p_{n}\right)=p_{i}$. Note that this mapping is surjective, continuous and open (the latter meaning that open sets of $Q^{n}$ are mapped onto open sets of $Q$ ).

2.1. Voronoi partitions. We present here some relevant concepts on Voronoi diagrams and refer the reader to [11, 23] for comprehensive treatments. A partition of $Q$ is a collection of $n$ polygons $\mathcal{W}=\left\{W_{1}, \ldots, W_{n}\right\}$ with disjoint interiors whose union is $Q$. Of course, more general types of partitions could be considered (as, for instance, continuous deformations of the previous ones), but these ones will be sufficient for our purposes. The Voronoi partition $\mathcal{V}(P)=\left(V_{1}(P), \ldots, V_{n}(P)\right)$ of $Q$ generated by the points $\left(p_{1}, \ldots, p_{n}\right)$ is defined by:

$$
V_{i}(P)=\left\{q \in Q \mid\left\|q-p_{i}\right\| \leq\left\|q-p_{j}\right\|, \forall j \neq i\right\} .
$$

For simplicity, we shall refer to $V_{i}(P)$ as $V_{i}$. Since $Q$ is a convex polygon, the boundary of each $V_{i}$ is the union of a finite number of segments. If $V_{i}$ and $V_{j}$ share an edge, i.e., $V_{i} \cap V_{j}$ is neither empty nor a singleton, then $p_{i}$ is called a (Voronoi) neighbor of $p_{j}$ (and vice-versa). All Voronoi neighboring relations are encoded in the mapping $\mathcal{N}: Q^{n} \times\{1, \ldots, n\} \rightarrow 2^{\{1, \ldots, n\}}$ where $\mathcal{N}(P, i)$ is the set of indexes of the Voronoi neighbors of $p_{i}$. Of course, $j \in \mathcal{N}(P, i)$ if and only if $i \in \mathcal{N}(P, j)$. We will often omit $P$ and instead write $\mathcal{N}(i)$.

For $P \in Q^{n}$, the vertexes of the Voronoi partition $\mathcal{V}(P)$ are classified as follows: the vertex $v$ is of type (a) if it is the center of the circle passing through three generators (say, $p_{i}, p_{j}$, and $p_{k}$ ), the vertex $v$ is of type (b) if it is the intersection between an edge of $Q$ and the bisector determined by two generators (say, $e, p_{i}$, and $p_{j}$ ), and the vertex $v$ is of type (c) if it is a vertex of $Q$, i.e., it is determined by two edges of $Q$ and by the generator of a cell containing it (say, $e, f$, and $p_{i}$ ). Correspondingly, we shall write $v(i, j, k), v(e, i, j)$, and $v(e, f, i)$ respectively, whenever we are interested in making explicit the elements defining the vertex $v$. The vertex $v \in \operatorname{Ve}\left(V_{i}(P)\right)$ is said to be nondegenerate if it is determined by exactly three elements (e.g., as described 
above, either three generators, or an edge and two generators, or two edges and one generator), otherwise it is said to be degenerate. Further, the configuration $P$ is said to be nondegenerate at the ith generator if all vertexes $v \in \mathrm{Ve}\left(V_{i}(P)\right)$ are nondegenerate, otherwise $P$ is degenerate at the ith generator. Finally, a configuration $P$ is said to be nondegenerate if all its vertexes are nondegenerate, otherwise it is said to be degenerate. These concepts are illustrated in Fig. [2.1]

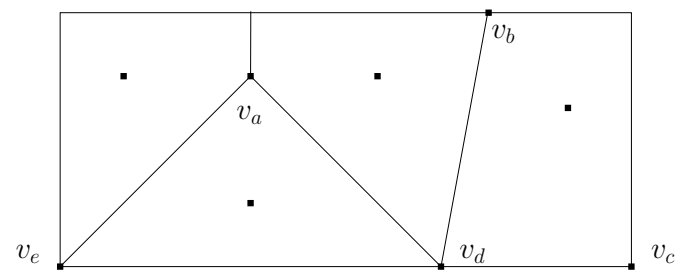

FIG. 2.1. A Voronoi partition with degenerate and nondegenerate vertexes. Vertexes $v_{a}, v_{b}$, and $v_{c}$ are nondegenerate vertexes of type $(a),(b),(c)$, respectively. Vertexes $v_{d}$ and $v_{e}$ are degenerate.

For $P \in Q^{n}$, the edges of the Voronoi partition $\mathcal{V}(P)$ are classified as follows: the edge $e$ is of type (a) if it is a segment of the bisector determined by two generators (say, $p_{i}, p_{j}$ ), the edge $e$ is of type (b) if it is contained in the boundary of $Q$, i.e., it is a subset of an edge of $Q$ and it belongs to a single cell (say, the cell of the generator $\left.p_{i}\right)$. Correspondingly, we shall write $e(i, j)$ and $e(i)$ respectively, whenever we are interested in making explicit the elements defining the edge $e$. Further, when considering an edge of type (a), we let $n_{e(i, j)}$ denote the unit normal to $e(i, j)$ pointing toward $\operatorname{int}\left(V_{i}(P)\right)$. When considering an edge of type (b), we let $n_{e(i)}$ denote the unit normal to $e(i)$ pointing toward $\operatorname{int}(Q)$.

2.2. The disk-covering and the sphere-packing problems. We are interested in the following locational optimization problems

$$
\begin{aligned}
& \min _{p_{1}, \ldots, p_{n}}\left\{\max _{q \in Q}\left\{\min _{i \in\{1, \ldots, n\}}\left\|q-p_{i}\right\|\right\}\right\}, \\
& \max _{p_{1}, \ldots, p_{n}}\left\{\min _{\substack{i, j \in\{1, \ldots, n\} \\
i \neq j, e \in \operatorname{Ed}(Q)}}\left\{\frac{1}{2}\left\|p_{i}-p_{j}\right\|, \mathrm{D}_{e}\left(p_{i}\right)\right\}\right\} .
\end{aligned}
$$

The optimization problem (2.1) is referred to as the $p$-center problem in [13, 29. Throughout the paper, we will refer to it as the multi-circumcenter problem. In the context of coverage control of mobile sensor networks [10, the multi-circumcenter problem corresponds to considering the worst case scenario, in which no information is available on the distribution of the events taking place in the environment $Q$. The network therefore tries to minimize the largest possible distance of any point in $Q$ to one of the generators' locations given by $p_{1}, \ldots, p_{n}$, i.e. to minimize the function,

$$
\mathcal{H}_{\mathrm{DC}}(P)=\max _{q \in Q}\left\{\min _{i \in\{1, \ldots, n\}}\left\|q-p_{i}\right\|\right\}=\max _{i \in\{1, \ldots, n\}}\left\{\max _{q \in V_{i}}\left\|q-p_{i}\right\|\right\} .
$$

It is conjectured in 29 that this problem can be restated as a disk-covering problem: how to cover a region with (possibly overlapping) disks of minimum radius. The disk-covering problem then reads:

$$
\min \left\{R \mid \cup_{i \in\{1, \ldots, n\}} \bar{B}_{2}\left(p_{i}, R\right) \supseteq Q\right\} .
$$


We shall present a proof of this statement in Theorem 4.7 below. Given a polytope $W$ in $\mathbb{R}^{N}$, its circumcenter, denoted by $\mathrm{CC}(W)$, is the center of the minimum-radius sphere that contains $W$. The circumradius of $W$, denoted by $\mathrm{CR}(W)$, is the radius of this sphere. We will say that $P$ is a circumcenter Voronoi configuration if $p_{i}=$ $\mathrm{CC}\left(V_{i}(P)\right)$, for all $i \in\{1, \ldots, n\}$. We denote by $\operatorname{Ve}_{\mathrm{DC}}(\mathcal{V}(P))$ the set of vertexes of the Voronoi partition where the value $\mathcal{H}_{\mathrm{DC}}(P)$ is attained, i.e. $v \in \operatorname{Ve}_{\mathrm{DC}}(\mathcal{V}(P))$ if there exists $i$ such that $v \in V_{i}(P)$ and $\left\|v-p_{i}\right\|=\mathcal{H}_{\mathrm{DC}}(P)$.

We will refer to the optimization problem (2.2) as the multi-incenter problem. In the context of applications, this problem corresponds to the situation where we are interested in maximizing the coverage of the area $Q$ in such a way that the sensing radius of the generators do not overlap (in order not to interfere with each other) or leave the environment. We therefore consider the maximization of the function

$$
\mathcal{H}_{\mathrm{SP}}(P)=\min _{\substack{i, j \in\{1, \ldots, n\} \\ i \neq j, e \in \operatorname{Ed}(Q)}}\left\{\frac{1}{2}\left\|p_{i}-p_{j}\right\|, \mathrm{D}_{e}\left(p_{i}\right)\right\}=\min _{i \in\{1, \ldots, n\}}\left\{\min _{q \notin \operatorname{int}\left(V_{i}\right)}\left\|q-p_{i}\right\|\right\} .
$$

A similar conjecture to the one presented above is that the multi-incenter problem can be restated as a sphere-packing problem: how to maximize the coverage of a region with non-overlapping disks (contained in the region) of minimum radius. The problem reads:

$$
\max \left\{R \mid \cup_{i \in\{1, \ldots, n\}} \bar{B}_{2}\left(p_{i}, R\right) \subseteq Q, B_{2}\left(p_{i}, R\right) \cap B_{2}\left(p_{j}, R\right)=\emptyset\right\} .
$$

In Theorem 4.8 we provide a positive answer to this question. Given a polytope $W$ in $\mathbb{R}^{N}$, its incenter set (or Chebyshev center set, see [6]), denoted by $\operatorname{IC}(W)$, is the set of the centers of maximum-radius spheres contained in $W$. The inradius of $W$, denoted by $\operatorname{IR}(W)$, is the common radius of these spheres. We will say that $P \in Q^{n}$ is an incenter Voronoi configuration if $p_{i} \in \operatorname{IC}\left(V_{i}(P)\right)$, for all $i \in\{1, \ldots, n\}$. If $P$ is an incenter Voronoi configuration, and each Voronoi region $V_{i}(P)$ has a unique incenter, $\operatorname{IC}\left(V_{i}(P)\right)=\left\{p_{i}\right\}$, then we will say that $P$ is a generic incenter Voronoi configuration. We denote by $\operatorname{Ed}_{\mathrm{SP}}(\mathcal{V}(P))$ the set of edges of the Voronoi partition where the value $\mathcal{H}_{\mathrm{SP}}(P)$ is attained, i.e. $e \in \operatorname{Ed}_{\mathrm{SP}}(\mathcal{V}(P))$ if there exists $i$ such that $e \in \operatorname{Ed}\left(V_{i}(P)\right)$ and $\mathrm{D}_{e}\left(p_{i}\right)=\mathcal{H}_{\mathrm{SP}}(P)$.

2.3. Nonsmooth analysis. The following facts on nonsmooth analysis [9] will be most helpful in analyzing the properties of the locational optimization functions for the disk-covering and the sphere-packing problems, as well as the convergence of the distributed algorithms we will propose to extremize them.

Definition 2.2. A function $f: \mathbb{R}^{N} \rightarrow \mathbb{R}$ is said to be locally Lipschitz near $x \in \mathbb{R}^{N}$ if there exist positive constants $L_{x}$ and $\epsilon$ such that $\left|f(y)-f\left(y^{\prime}\right)\right| \leq L_{x}\left\|y-y^{\prime}\right\|$ for all $y, y^{\prime} \in B_{N}(x, \epsilon)$.

Note that continuously differentiable functions at $x$ are locally Lipschitz near $x$. The usual right directional derivative of $f$ at $x$ in the direction of $v \in \mathbb{R}^{N}$ is defined as

$$
f^{\prime}(x, v)=\lim _{t \rightarrow 0^{+}} \frac{f(x+t v)-f(x)}{t},
$$

when this limits exists. On the other hand, the generalized directional derivative of $f$ at $x$ in the direction of $v \in \mathbb{R}^{N}$ is defined as

$$
f^{o}(x ; v)=\limsup _{\substack{y \rightarrow x \\ t \rightarrow 0^{+}}} \frac{f(y+t v)-f(y)}{t} .
$$


This notion of directional derivative has the advantage of always being well-defined.

Definition 2.3. A function $f: \mathbb{R}^{N} \rightarrow \mathbb{R}$ is said to be regular at $x \in \mathbb{R}^{N}$ if for all $v \in \mathbb{R}^{N}, f^{\prime}(x ; v)$ exists and $f^{o}(x ; v)=f^{\prime}(x ; v)$.

Again, a continuously differentiable function at $x$ is regular at $x$. Also, a locally Lipschitz function at $x$ which is convex (or concave) is also regular (cf. Proposition 2.3.6 in 9]).

From Rademacher's Theorem 9], we know that locally Lipschitz functions are continuously differentiable almost everywhere (in the sense of Lebesgue measure). If $\Omega_{f}$ denotes the set of points in $\mathbb{R}^{N}$ at which $f$ fails to be differentiable, and $S$ denotes any other set of measure zero, the generalized gradient of $f$ is defined by

$$
\partial f(x)=\operatorname{co}\left\{\lim _{i \rightarrow+\infty} d f\left(x_{i}\right) \mid x_{i} \rightarrow x, x_{i} \notin S \cup \Omega_{f}\right\} .
$$

Note that this definition coincides with $d f(x)$ if $f$ is continuously differentiable at $x$. The generalized gradient and the generalized directional derivative (cf. Proposition 2.1.2 in 9]) are related by $f^{o}(x ; v)=\max \{\zeta \cdot v \mid \zeta \in \partial f(x)\}$, for each $v \in \mathbb{R}^{N}$. A point $x \in \mathbb{R}^{N}$ which verifies that $0 \in \partial f(x)$ is called a critical point of $f$.

The following result corresponds to Proposition 2.3.12 in [9].

Proposition 2.4. Let $\left\{f_{k}: \mathbb{R}^{N} \rightarrow \mathbb{R} \mid k \in\{1, \ldots, m\}\right\}$ be a finite collection of locally Lipschitz functions near $x \in \mathbb{R}^{N}$. Consider $f\left(x^{\prime}\right)=\min \left\{f_{k}\left(x^{\prime}\right) \mid k \in\{1, \ldots, m\}\right\}$. Then,

(i) $f$ is locally Lipschitz near $x$,

(ii) if $I\left(x^{\prime}\right)$ denotes the set of indexes $k$ for which $f_{k}\left(x^{\prime}\right)=f\left(x^{\prime}\right)$, we have,

$$
\partial f(x) \subset \operatorname{co}\left\{\partial f_{i}(x) \mid i \in I(x)\right\},
$$

and if each $f_{i}$ is regular at $x$ for $i \in I(x)$, then equality holds and $f$ is regular at $x$.

The extrema of Lipschitz functions are characterized by the following result.

Proposition 2.5. Let $f$ be a locally Lipschitz function at $x \in \mathbb{R}^{N}$. If $f$ attains a local minimum or maximum at $x$, then $0 \in \partial f(x)$, i.e., $x$ is a critical point.

Let Ln $: 2^{\mathbb{R}^{N}} \rightarrow 2^{\mathbb{R}^{N}}$ be the set-valued mapping that associates to each subset $S$ of $\mathbb{R}^{N}$ the set of its least-norm elements $\operatorname{Ln}(S)$. If the set $S$ is convex, then the set $\operatorname{Ln}(S)$ reduces to a singleton and we note the equivalence $\operatorname{Ln}(S)=\operatorname{proj}_{S}(0)$. Along the paper, we shall only apply this function to convex sets. For a locally Lipschitz function $f$, we consider the generalized gradient vector field $\operatorname{Ln}(\partial f): \mathbb{R}^{N} \rightarrow \mathbb{R}^{N}$ given by $x \mapsto \operatorname{Ln}(\partial f)(x)=\operatorname{Ln}(\partial f(x))$.

Theorem 2.6. Let $f$ be a locally Lipschitz function at $x$. Assume $0 \notin \partial f(x)$. Then, there exists $T>0$ such that

$$
f(x-t \operatorname{Ln}(\partial f)(x)) \leq f(x)-\frac{t}{2}\|\operatorname{Ln}(\partial f)(x)\|^{2}, \quad 0<t<T .
$$

The vector $-\operatorname{Ln}(\partial f)(x)$ is called a direction of descent.

2.4. Stability analysis via nonsmooth Lyapunov functions. For differential equations with discontinuous right-hand sides, solutions are defined in terms of differential inclusions [15.

Let $F: \mathbb{R}^{N} \rightarrow 2^{\mathbb{R}^{N}}$ be a set-valued map. Consider the differential inclusion

$$
\dot{x} \in F(x) .
$$


A solution to this equation on an interval $\left[t_{0}, t_{1}\right] \subset \mathbb{R}$ is defined as an absolutely continuous function $x:\left[t_{0}, t_{1}\right] \rightarrow \mathbb{R}^{N}$ such that $\dot{x}(t) \in F(x(t))$ for almost all $t \in$ $\left[t_{0}, t_{1}\right]$. Given $x_{0} \in \mathbb{R}^{N}$, the existence of at least a solution with initial condition $x_{0}$ is guaranteed by the following lemma.

LEMMA 2.7. Let the mapping $F$ be upper semicontinuous with nonempty, compact and convex values. Then, given $x_{0} \in \mathbb{R}^{N}$, there exists at least a solution of (2.4) with initial condition $x_{0}$.

Now, consider the differential equation

$$
\dot{x}(t)=X(x(t)),
$$

where $X: \mathbb{R}^{N} \rightarrow \mathbb{R}^{N}$ is measurable and essentially locally bounded. The solution of this equation has to be understood in the Filippov sense. For each $x \in \mathbb{R}^{N}$, consider the set

$$
K[X](x)=\bigcap_{\delta>0} \bigcap_{\mu(S)=0} \operatorname{co}\left\{X\left(B_{N}(x, \delta) \backslash S\right)\right\},
$$

where $\mu$ denotes the usual Lebesgue measure in $\mathbb{R}^{N}$. Alternatively, one can show 25] that there exists a set $S_{X}$ of measure zero such that

$$
K[X](x)=\operatorname{co}\left\{\lim _{i \rightarrow+\infty} X\left(x_{i}\right) \mid x_{i} \rightarrow x, x_{i} \notin S \cup S_{X}\right\},
$$

where $S$ is any set of measure zero. A Filippov solution of (2.5) on an interval $\left[t_{0}, t_{1}\right] \subset \mathbb{R}$ is defined as a solution of the differential inclusion

$$
\dot{x} \in K[X](x) .
$$

Since the multivalued mapping $K[X]: \mathbb{R}^{N} \rightarrow 2^{\mathbb{R}^{N}}$ is upper semicontinuous with nonempty, compact, convex values and locally bounded (cf. [15]), the existence of Filippov solutions of (2.5) is guaranteed by Lemma 2.7

A set $M$ is weakly invariant (respectively strongly invariant) for (2.5) if for each $x_{0} \in M, M$ contains a maximal solution (respectively all maximal solutions) of (2.5). Given a locally Lipschitz function $f: \mathbb{R}^{N} \rightarrow \mathbb{R}$, the set-valued Lie derivative of $f$ with respect to $X$ at $x$ is defined as

$$
\widetilde{\mathcal{L}}_{X} f(x)=\{a \in \mathbb{R} \mid \exists v \in K[X](x) \text { such that } \zeta \cdot v=a, \forall \zeta \in \partial f(x)\} .
$$

For each $x \in \mathbb{R}^{N}, \widetilde{\mathcal{L}}_{X} f(x)$ is a closed and bounded interval in $\mathbb{R}$, possibly empty. If $f$ is continuously differentiable at $x$, then $\widetilde{\mathcal{L}}_{X} f(x)=\{d f \cdot v \mid v \in K[X](x)\}$. If, in addition, $X$ is continuous at $x$, then $\widetilde{\mathcal{L}}_{X} f(x)$ corresponds to the singleton $\left\{\mathcal{L}_{X} f(x)\right\}$, the usual Lie derivative of $f$ in the direction of $X$ at $x$. The importance of the set-valued Lie derivative stems from the next result 3 .

TheOREM 2.8. Let $x:\left[t_{0}, t_{1}\right] \rightarrow \mathbb{R}^{N}$ be a Filippov solution of (2.5). Let $f$ be a locally Lipschitz and regular function. Then $\frac{d}{d t}(f(x(t)))$ exists a.e. and $\frac{d}{d t}(f(x(t))) \in$ $\widetilde{\mathcal{L}}_{X} f(x(t))$ a.e.

The following result is a generalization of LaSalle principle for differential equations of the form (2.5) with nonsmooth Lyapunov functions. The formulation is taken from 3 , and slightly generalizes the one presented in [27].

ThEOREM 2.9 (LaSalle principle). Let $f: \mathbb{R}^{N} \rightarrow \mathbb{R}$ be a locally Lipschitz and regular function. Let $x_{0} \in \mathbb{R}^{N}$ and let $f^{-1}\left(\leq f\left(x_{0}\right), x_{0}\right)$ be the connected component of 
$\left\{x \in \mathbb{R}^{N} \mid f(x) \leq f\left(x_{0}\right)\right\}$ containing $x_{0}$. Assume the set $f^{-1}\left(\leq f\left(x_{0}\right), x_{0}\right)$ is bounded and assume either max $\widetilde{\mathcal{L}}_{X} f(x) \leq 0$ or $\widetilde{\mathcal{L}}_{X} f(x)=\emptyset$ for all $x \in f^{-1}\left(\leq f\left(x_{0}\right), x_{0}\right)$. Then $f^{-1}\left(\leq f\left(x_{0}\right), x_{0}\right)$ is strongly invariant for 2.5.

$$
Z_{X, f}=\left\{x \in \mathbb{R}^{N} \mid 0 \in \widetilde{\mathcal{L}}_{X} f(x)\right\} .
$$

Then, any solution $x:\left[t_{0},+\infty\right) \rightarrow \mathbb{R}^{N}$ of (2.5) starting from $x_{0}$ converges to the largest weakly invariant set $M$ contained in $\bar{Z}_{X, f} \cap f^{-1}\left(\leq f\left(x_{0}\right), x_{0}\right)$. Furthermore, if the set $M$ is a finite collection of points, then the limit of all solutions starting at $x_{0}$ exists and equals one of them.

The proof of the last fact in the theorem statement is the same as in the smooth case, since it only relies on the continuity of the trajectory. The next statement is based on Theorem 2 of [25].

Proposition 2.10. Under the same assumptions of Theorem[2.9 if $\max \widetilde{\mathcal{L}}_{X} f(x)<$ $-\epsilon<0$ a.e. on $\mathbb{R}^{N} \backslash Z_{X, f}$, then $Z_{X, f}$ is attained in finite time.

Proof. Let $x:\left[t_{0},+\infty\right) \rightarrow \mathbb{R}^{N}$ be a Filippov solution starting from $x_{0}$. We argue that there must exist $T$ such that $x(T) \in Z_{X, f}$. Otherwise, we have

$$
f(x(t))=f\left(x\left(t_{0}\right)\right)+\int_{t_{0}}^{t} \frac{d}{d s} f(x(s)) d s<f\left(x\left(t_{0}\right)\right)-\epsilon\left(t-t_{0}\right) \stackrel{t \rightarrow+\infty}{\longrightarrow}-\infty,
$$

which contradicts the fact that $f^{-1}\left(\leq f\left(x_{0}\right), x_{0}\right)$ is strongly invariant and bounded.

2.5. Nonsmooth gradient flows. Finally, we are in a position to present the nonsmooth analogue of well-known results on gradient flows. Given a locally Lipschitz and regular function $f$, consider the following generalized gradient flow

$$
\dot{x}(t)=-\operatorname{Ln}(\partial f)(x(t)) .
$$

Theorem 2.6 guarantees that, unless the flow is at a critical point, $-\operatorname{Ln}(\partial f)(x)$ is always a direction of descent at $x$. In general, the vector field $\operatorname{Ln}(\partial f)$ in (2.7) is discontinuous, and therefore its solution must be understood in the Filippov sense. Note that, since $f$ is locally Lipschitz, $\operatorname{Ln}(\partial f)=d f$ almost everywhere. An important observation in this setting is that $K[d f](x)=\partial f(x)$ (cf. [25]). The following result, which is a generalization of the discussion in [3], guarantees the convergence of this flow to the set of critical points of $f$.

Proposition 2.11. Let $x_{0} \in \mathbb{R}^{N}$ and assume $f^{-1}\left(\leq f\left(x_{0}\right), x_{0}\right)$ is bounded. Then, any solution $x:\left[t_{0},+\infty\right) \rightarrow \mathbb{R}^{N}$ of eq. (2.7] starting from $x_{0}$ converges asymptotically to the set of critical points of $f$ contained in $f^{-1}\left(\leq f\left(x_{0}\right), x_{0}\right)$.

Proof. Let $a \in \widetilde{\mathcal{L}}_{-\operatorname{Ln}(\partial f)} f(x)$. By definition, there exists $w \in K[-\operatorname{Ln}(\partial f)](x)=$ $-\partial f(x)$ such that $a=w \cdot \zeta$ for all $\zeta \in \partial f(x)$. In particular, for $\zeta=-w \in \partial f(x)$, we have $a=-\|w\|^{2} \leq 0$. Therefore, $\max \widetilde{\mathcal{L}}_{-\operatorname{Ln}(\partial f)} f(x) \leq 0$ or $\widetilde{\mathcal{L}}_{-\operatorname{Ln}(\partial f)} f(x)=\emptyset$. Now, resorting to the LaSalle principle (Theorem [2.9]), we deduce that any solution $x:\left[t_{0},+\infty\right) \rightarrow \mathbb{R}^{N}$ starting from $x_{0}$ converges to the largest weakly invariant set contained in $\bar{Z}_{-\operatorname{Ln}(\partial f), f} \cap f^{-1}\left(\leq f\left(x_{0}\right), x_{0}\right)$. Let us see that $Z_{-\operatorname{Ln}(\partial f), f}$ is equal to $L_{0}=\left\{x \in Q^{n} \mid 0 \in \partial f(x)\right\}$. Obviously, $L_{0} \subset Z_{-\operatorname{Ln}(\partial f), f \text {. Conversely, assume }}$

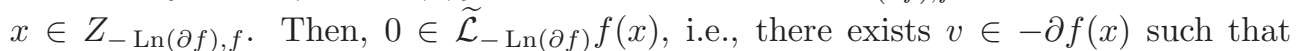
$\zeta \cdot v=0$ for all $\zeta \in \partial f(x)$. In particular, for $\zeta=-v$, we get $\|v\|^{2}=0$, that is, $v=0 \in \partial f(x)$, as desired. Note that $Z_{-\operatorname{Ln}(\partial f), f}=L_{0}$ is the equilibrium set 
of (2.7) and therefore is weakly invariant. Finally, we prove that it is also closed. Let $x \in \bar{Z}_{-\operatorname{Ln}(\partial f), f}$ and consider a sequence $\left\{x_{k} \in \mathbb{R}^{N} \mid k \in \mathbb{N}\right\} \subset Z_{-\operatorname{Ln}(\partial f), f}$ such that $x_{k} \rightarrow x$. Then, using the fact that the multivalued mapping $K[-v]$ is upper semicontinuous, for any $\epsilon>0$, there exists $k_{0}$ such that for $k \geq k_{0}, \partial f\left(x_{k}\right) \subset \partial f(x)+$ $B_{N}(0, \epsilon)$. Since $x_{k} \in Z_{-\operatorname{Ln}(\partial f), f}$, then $0 \in \partial f(x)+B_{N}(0, \epsilon)$ for all $\epsilon>0$, and this implies that $0 \in \partial f(x)$, i.e., $x \in Z_{-\operatorname{Ln}(\partial f), f}$. Hence the largest weakly invariant set contained in $\bar{Z}_{-\operatorname{Ln}(\partial f), f} \cap f^{-1}\left(\leq f\left(x_{0}\right), x_{0}\right)$ is $Z_{-\operatorname{Ln}(\partial f), f} \cap f^{-1}\left(\leq f\left(x_{0}\right), x_{0}\right)=$ $\left\{x \in f^{-1}\left(\leq f\left(x_{0}\right), x_{0}\right) \mid 0 \in \partial f(x)\right\}$.

3. The 1-center problems. In this section we consider the disk-covering and the sphere-packing problems with a single generator, i.e., $n=1$. This treatment will give us the necessary insight to tackle later the more involved multi-center version of both problems. When $n=1$, the minimization of $\mathcal{H}_{\mathrm{DC}}$ simply consists of finding the center of the minimum-radius sphere enclosing the polygon $Q$. On the other hand, the maximization of $\mathcal{H}_{\mathrm{SP}}$ corresponds to determining the center of the maximum-radius sphere contained in $Q$. Let us therefore define the functions

$$
\begin{aligned}
\lg _{Q}(p) & =\max \{\|q-p\| \mid q \in Q\}=\max \{\|v-p\| \mid v \in \mathrm{Ve}(Q)\}, \\
\operatorname{sm}_{Q}(p) & =\min \{\|q-p\| \mid q \notin \operatorname{int}(Q)\}=\min \left\{\mathrm{D}_{e}(p) \mid e \in \operatorname{Ed}(Q)\right\} .
\end{aligned}
$$

When $n=1$, we then have that $\mathcal{H}_{\mathrm{DC}}=\lg _{Q}: Q \rightarrow \mathbb{R}$ and $\mathcal{H}_{\mathrm{SP}}=\operatorname{sm}_{Q}: Q \rightarrow \mathbb{R}$.

3.1. Smoothness and critical points. We here discuss the smoothness properties and the critical points of the 1-center functions. Since the function $\lg _{Q}$ is the maximum of a (finite) set of convex functions in $p$, it is also a convex function [6]. Therefore, any local minimum of $\lg _{Q}$ is also global.

LEMma 3.1. The function $\lg _{Q}$ has a unique global minimum, which is the circumcenter of the polygon $Q$.

Proof. Let $F: \mathbb{R} \rightarrow \mathbb{R}$ be any continuous non-decreasing function. Then,

$$
F\left(\lg _{Q}(p)\right)=\max \{F(\|v-p\|) \mid v \in \operatorname{Ve}(Q)\} .
$$

If we take $F(x)=x^{2}$, each function $\|v-p\|^{2}$ is strictly convex, and hence $F\left(\lg _{Q}(p)\right)$ is also strictly convex. Therefore, this latter function has a single minimum on $Q$. Since any global minimum of $\lg _{Q}$ is also a global minimum of $F\left(\lg _{Q}(p)\right)$, we conclude the result.

The function $\operatorname{sm}_{Q}$ is the minimum of a (finite) set of affine (hence, concave) functions defined on the half-planes determined by the edges of $Q$, and hence it is also a concave function [6] on the intersection of their domains, which is precisely $Q$. Therefore, any local maximum of $\operatorname{sm}_{Q}$ is also global. However, this maximum is not unique in general.

LEMma 3.2. The incenter set of the polygon $Q$ is the set of maxima of the function $\operatorname{sm}_{Q}$ and it is a segment.

Proof. It is clear that the set of maxima of $\operatorname{sm}_{Q}$ is $\operatorname{IC}(Q)$. As a consequence of the concavity of $\operatorname{sm}_{Q}$ over the convex domain $Q$, one deduces that $\operatorname{IC}(Q)$ is a convex set. Now, assume there are three points $p_{1}, p_{2}, p_{3}$ in $\operatorname{IC}(Q)$ which are not aligned. Since $B_{2}(q, \operatorname{IR}(Q)) \subset Q$ for all $q \in \operatorname{co}\left(p_{1}, p_{2}, p_{3}\right) \subset \operatorname{IC}(Q)$, and $\operatorname{co}\left(p_{1}, p_{2}, p_{3}\right)$ has nonempty interior, there exist $q_{0} \in Q$ and $r>\operatorname{IR}(Q)$ such that $B_{2}\left(q_{0}, r\right) \subset Q$, which is a contradiction.

Note that the circumcenter of a polygon can be computed via the finite-step algorithm described in [28]. The incenter set of a polygon can be computed via the 
following linear program: maximize the inradius subject to the constraints that the distance between the incenter and each of the polygon edges must be greater than or equal to the inradius. In what follows, let us examine dynamical systems that compute these geometric centers.

Proposition 3.3. The functions $\lg _{Q}(p), \operatorname{sm}_{Q}(p)$ are locally Lipschitz and regular, and their generalized gradients are given by

$$
\begin{aligned}
\partial \lg _{Q}(p) & =\operatorname{co}\left\{\operatorname{vrs}(p-v) \mid v \in \operatorname{Ve}(Q), \lg _{Q}(p)=\|p-v\|\right\}, \\
\partial \operatorname{sm}_{Q}(p) & =\operatorname{co}\left\{n_{e} \mid e \in \operatorname{Ed}(Q), \operatorname{sm}_{Q}(p)=\mathrm{D}_{e}(p)\right\} .
\end{aligned}
$$

Moreover,

$$
0 \in \partial \lg _{Q}(p) \Longleftrightarrow p=\mathrm{CC}(Q), \quad 0 \in \partial \operatorname{sm}_{Q}(p) \Longleftrightarrow p \in \operatorname{IC}(Q),
$$

and, if $0 \in \operatorname{int}\left(\partial \operatorname{sm}_{Q}(p)\right)$, then $\operatorname{IC}(Q)=\{p\}$.

Proof. Given the expressions in (3.1) and Proposition 2.4 we deduce that $\lg _{Q}$ and $\operatorname{sm}_{Q}$ are locally Lipschitz and regular, and that their generalized gradients are respectively given by (3.2) and (3.3.). Concerning (3.4), the implications from right to left in (3.4) readily follow from Proposition 2.5. As for the other ones, note that it is sufficient to prove that $p$ is a local minimum, respectively that $p$ is a local maximum. We prove the result for the function $\lg _{Q}$. The proof for $\operatorname{sm}_{Q}$ is analogous. Assume that $0 \in \partial \lg _{Q}(p)$. Then, there exist vertexes $v_{i_{1}}, \ldots, v_{i_{K}}$ of $Q$ with $\lg _{Q}(p)=\left\|v_{i_{l}}-p\right\|$, $l \in\{1, \ldots, K\}$ such that $0=\sum_{l \in\{1, \ldots, K\}} \lambda_{l} \operatorname{vrs}\left(p-v_{i_{l}}\right)$, where $\sum_{l \in\{1, \ldots, K\}} \lambda_{l}=1$, $\lambda_{l} \geq 0, l \in\{1, \ldots, K\}$. Let $U$ be a neighborhood of $p$ and take $q \in U$. One can show that there must exist $l^{*}$ such that $\left(p-v_{i_{l^{*}}}\right) \cdot(q-p) \geq 0$, since otherwise $0=0 \cdot(q-p)=\left(\sum_{l \in\{1, \ldots, K\}} \lambda_{l} \operatorname{vrs}\left(p-v_{i_{l}}\right)\right) \cdot(q-p)<0$, which is a contradiction. Then,

$$
\left\|q-v_{i_{l^{*}}}\right\|^{2}=\|q-p\|^{2}+\left\|p-v_{i_{l^{*}}}\right\|^{2}-2(q-p) \cdot\left(v_{i_{l^{*}}}-p\right) \geq\left\|p-v_{i_{l^{*}}}\right\|^{2} .
$$

Therefore, $\lg _{Q}(q) \geq\left\|p-v_{i_{l^{*}}}\right\|=\lg _{Q}(p)$, which shows that $p$ is a local minimum. Finally, if $0 \in \operatorname{int}\left(\partial \operatorname{sm}_{Q}(p)\right)$, then one can see that $p$ is a strict local maximum. Furthermore, there cannot be any other local (hence global) maximum of $\mathrm{sm}_{Q}$, as we now show: assume $\bar{p} \in \mathrm{IC}(Q)$. By hypothesis, the sphere $B_{2}\left(\bar{p}, \operatorname{sm}_{Q}(p)\right)$ centered at $\bar{p}$ of radius $\operatorname{sm}_{Q}(p)$ is contained in $Q$. Consider the vector $\bar{p}-p$. By Lemma 2.1 there exists $e \in \operatorname{Ed}(Q)$ with $\mathrm{D}_{e}(p)=\operatorname{sm}_{Q}(p)$ such that $(\bar{p}-p) \cdot n_{e}>0$. Therefore, there are points of $B_{2}\left(\bar{p}, \operatorname{sm}_{Q}(p)\right)$ which necessarily belong to the half-plane defined by $e$ where $Q$ is not contained, which is a contradiction.

3.2. Convergence properties for nonsmooth gradient flows. Here we study the generalized gradient flows arising from the two 1-center functions. An immediate consequence of Propositions 2.11 and 3.3 is the following result.

Corollary 3.4. The gradient flows of the functions $\lg _{Q}$ and $\operatorname{sm}_{Q}$

$$
\begin{aligned}
& \dot{x}(t)=-\operatorname{Ln}\left(\partial \lg _{Q}\right)(x(t)), \\
& \dot{x}(t)=\operatorname{Ln}\left(\partial \operatorname{sm}_{Q}\right)(x(t)),
\end{aligned}
$$

converge asymptotically to the circumcenter $\operatorname{CC}(Q)$ and the incenter set $\operatorname{IC}(Q)$, respectively.

The following two propositions discuss the convergence properties of the gradient descents.

Proposition 3.5. If $0 \in \operatorname{int}\left(\partial \lg _{Q}(\mathrm{CC}(Q))\right)$, then the flow reaches $\mathrm{CC}(Q)$ in finite time. 
Proof. Let us prove that there exists $\epsilon>0$ such that $\max \widetilde{\mathcal{L}}_{-\operatorname{Ln}\left[\lg _{Q}\right]} \lg _{Q}<-\epsilon$ a.e. on $Q \backslash\{\mathrm{CC}(Q)\}$. Take $p \neq \mathrm{CC}(Q)$. We know that each element $a \in \widetilde{\mathcal{L}}_{-\operatorname{Ln}\left[\lg _{Q}\right]} \lg _{Q}(p)$ can be expressed as $a=-\|w\|^{2}$, with $-w \in \partial \lg _{Q}(p)$. Therefore, we have

$$
\max \widetilde{\mathcal{L}}_{-\operatorname{Ln}\left[\lg _{Q}\right]} \lg _{Q}(p)=-\left\|\operatorname{Ln}\left[\lg _{Q}\right](p)\right\|^{2} .
$$

If there is a single vertex of $Q$ involved in $\partial \lg _{Q}(p)$, then moving along the direction $-\operatorname{Ln}\left[\lg _{Q}\right](p)$ obviously decreases the distance to that vertex while maintaining constant the norm of the least-norm element, which is 1 . If there are two or more vertexes involved, from the expression for the generalized gradient at $p$ (cf. eq. (3.2)), it is clear that one can express it as

$$
\left\{x \in \mathbb{R}^{N} \mid g_{1}(x) \leq 0, \ldots, g_{s}(x) \leq 0\right\},
$$

for some linear functions $g_{r}$. Note that the points $x \in \partial \lg _{Q}(p)$ such that $g_{r}(x)=0$ for some $r$ correspond to a set of the form $\operatorname{co}\left\{\operatorname{vrs}\left(p-v_{r, 1}\right), \operatorname{vrs}\left(p-v_{r, 2}\right)\right\}$, for certain vertexes $v_{r, 1}, v_{r, 2}$ of $Q$. Now, the computation of the least-norm element in $\partial \lg _{Q}(p)$ can be formulated as the convex problem,

$$
\begin{aligned}
& \text { minimize }\|x\|^{2} \\
& \text { subject to } g_{1}(x) \leq 0, \ldots, g_{s}(x) \leq 0 .
\end{aligned}
$$

Let $x^{*}=\operatorname{Ln}\left[\lg _{Q}\right](p)$. Let $R$ denote the set of indexes $r$ for which $g_{r}\left(x^{*}\right)=0$. Then $x^{*}$ is a regular point 22, meaning to say that $d g_{r}\left(x^{*}\right), r \in R$ are linearly independent vectors. This is because the cardinality of $R$ is at most 2 (since the intersection of two lines already determines a point), and the gradients of the functions $g_{r}$ are independent when considered pairwise. We apply then the Kuhn-Tucker first-order necessary conditions for optimality 22 to conclude that there must exist $r^{*} \in R$ such that $g_{r^{*}}\left(x^{*}\right)=0$. It is easy to see that $r^{*}$ must be unique, since otherwise $x^{*}$ does not have minimum norm. Therefore, we have that $\operatorname{Ln}\left[\lg _{Q}\right](p)$ is determined as the least-norm element in $\operatorname{co}\left\{\operatorname{vrs}\left(p-v_{r^{*}, 1}\right), \operatorname{vrs}\left(p-v_{r^{*}, 2}\right)\right\}$. As a consequence, moving along the direction $-\operatorname{Ln}\left[\lg _{Q}\right](p)$ decreases the distance to the vertexes $v_{r^{*}, 1}, v_{r^{*}, 2}$, and hence the norm of the least-norm element decreases. If, along the flow (3.5), a new vertex of $Q$ enters in the computation of $\partial \lg _{Q}(p(t))$, then there can be a jump in the norm of $\operatorname{Ln}\left[\lg _{Q}\right](p(t))$, which by definition will always be decreasing. Finally, note that if $v_{r^{*}, 1}, v_{r^{*}, 2}$ are active at the circumcenter, then they cannot be opposite with respect to $\mathrm{CC}(Q)$ precisely because of the assumption that 0 lies in $\operatorname{int}\left(\partial \lg _{Q}(\mathrm{CC}(Q))\right)$. Therefore, we conclude

$$
\begin{gathered}
\left\|\operatorname{Ln}\left[\lg _{Q}\right](p)\right\| \geq \epsilon=\min \{1,\{\|\operatorname{Ln}(\operatorname{co}\{\operatorname{vrs}(\operatorname{CC}(Q)-v), \operatorname{vrs}(\operatorname{CC}(Q)-w)\})\| \mid \\
v, w \in I(\operatorname{CC}(Q)), \operatorname{CC}(Q)-v \neq-(\operatorname{CC}(Q)-w)\}\}>0, \quad \forall p \neq \operatorname{CC}(Q) .
\end{gathered}
$$

Resorting now to Proposition 2.10 we deduce that the circumcenter $\operatorname{CC}(Q)$ is attained in finite time.

REMARK 3.6. Note that if $0 \in \partial \lg _{Q}(\mathrm{CC}(Q)) \backslash \operatorname{int}\left(\partial \lg _{Q}(\mathrm{CC}(Q))\right)$, then generically convergence is achieved over an infinite time horizon.

Proposition 3.7. The flow (3.6) reaches the set $\mathrm{IC}(Q)$ in finite time.

Proof. Let $p \notin \operatorname{IC}(Q)$. We know $\min \widetilde{\mathcal{L}}_{\operatorname{Ln}\left[\operatorname{sm}_{Q}\right]} \operatorname{sm}_{Q}(p)=\left\|\operatorname{Ln}\left[\operatorname{sm}_{Q}\right](p)\right\|^{2}$. Moreover, for all $p \notin \operatorname{IC}(Q)$, we have

$$
\left\|\operatorname{Ln}\left[\operatorname{sm}_{Q}\right](p)\right\| \geq \epsilon=\min \left\{1,\left\{\left\|\operatorname{Ln}\left(\operatorname{co}\left\{n_{e}, n_{f}\right\}\right)\right\| \mid e, f \in \operatorname{Ed}(Q), n_{e} \neq-n_{f}\right\}\right\}>0 .
$$


Resorting to Proposition 2.10 we deduce the desired result.

Fig. 3.1] shows an example of the implementation of the gradient descent (3.5) and (3.6). Note that if the circumcenter $\operatorname{CC}(Q)$ (respectively the incenter set $\operatorname{IC}(Q)$ ) is first computed offline, then the strategy of directly going toward it would converge in a less "erratic" way. Note also that the move-toward-the-center strategy is exponentially fast.
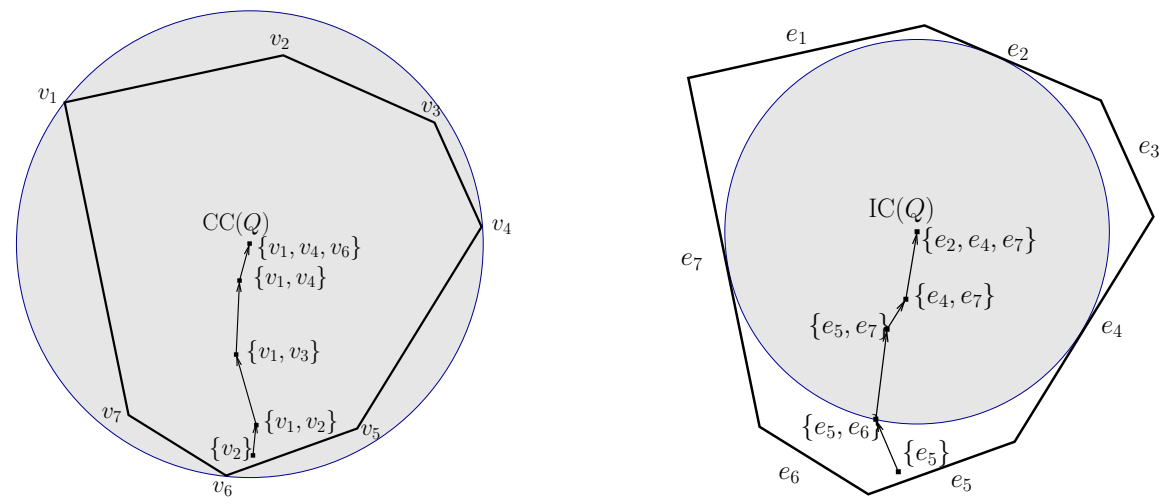

FIG. 3.1. Illustration of the gradient descent of $\lg _{Q}$ and $\operatorname{sm}_{Q}$. The points where the curve $t \mapsto p(t)$ fails to be differentiable correspond to points where there is a new vertex $v$ of $Q$ such that $\|p(t)-v\|=\lg _{Q}(p(t))$ (respectively a new edge e of $Q$ such that $\mathrm{D}_{e}(p(t))=\operatorname{sm}_{Q}(p(t))$ ). The circumcenter and the incenter are attained in finite time according to Propositions [3.5 and 3.7

Finally, we conclude this section with four results useful for later developments.

Lemma 3.8. Let $q \in Q$, let $v(q)$ be one of the vertexes of $Q$ which is furthest away from $q$, and let $e(q)$ be one of the edges of $Q$ which is nearest to $q$. Then,

(i) $\operatorname{Ln}\left[\lg _{Q}\right](q) \cdot(q-v(q)) \geq 0$, and the inequality is strict if $q \neq \mathrm{CC}(Q)$,

(ii) $(q-\mathrm{CC}(Q)) \cdot(q-v(q)) \geq\|q-\mathrm{CC}(Q)\|^{2} / 2$,

(iii) $\operatorname{Ln}\left[\operatorname{sm}_{Q}\right](q) \cdot n_{e} \geq 0$, and the inequality is strict if $q \notin \operatorname{IC}(Q)$, and

(iv) $(x-q) \cdot n_{e} \geq \operatorname{IR}(Q)-\mathrm{D}_{e}(q) \geq 0$ for any $x \in \operatorname{IC}(Q)$, and the second inequality is strict if $q \notin \mathrm{IC}(Q)$.

Proof. Let $q$ be a point in $Q$. If $q=\mathrm{CC}(Q)$, claims (i) and (ii) are obviously satisfied since $\operatorname{Ln}\left[\lg _{Q}\right](q)=0$. Assume then that $q \neq \mathrm{CC}(Q)$. Let us prove first (i). By Proposition $3.30 \notin \partial \lg _{Q}(q)$, and hence $\operatorname{Ln}\left[\lg _{Q}\right](q) \neq 0$. Let us prove $\operatorname{Ln}\left[\lg _{Q}\right](q) \cdot(q-v(q))>0$ reasoning by contradiction. If $\operatorname{Ln}\left[\lg _{Q}\right](q) \cdot(q-v(q)) \leq 0$, then $d / d t\left(\left\|q-t \operatorname{Ln}\left[\lg _{Q}\right](q)-v\right\|\right)_{t=0}=\operatorname{vrs}(q-v) \cdot\left(-\operatorname{Ln}\left[\lg _{Q}\right](q)\right) \geq 0$, which implies that $\left\|q-t \operatorname{Ln}\left[\lg _{Q}\right](q)-v\right\| \geq\|q-v\|=\lg _{Q}(q)$ for $t>0$ small enough. On the other hand, invoking Theorem [2.6] we have that $\lg _{Q}(q)-t\left\|\operatorname{Ln}\left[\lg _{Q}\right](q)\right\|^{2} / 2 \geq$ $\lg _{Q}\left(q-t \operatorname{Ln}\left[\lg _{Q}\right](q)\right) \geq\left\|q-t \operatorname{Ln}\left[\lg _{Q}\right](q)-v\right\|$. Gathering both facts, we conclude $-t\left\|\operatorname{Ln}\left[\lg _{Q}\right](q)\right\|^{2} / 2 \geq 0$, which is a contradiction.

Let us now prove (ii). Since $q \neq \operatorname{CC}(Q)$, we have $\|q-v(q)\|>\operatorname{CR}(Q)$. Consider then a ball $\bar{B}_{2}(v(q),\|q-v(q)\|)$ centered at the vertex $v(q)$, with radius $\|q-v(q)\|$. By definition of the circumcenter, $\operatorname{CC}(Q)$ must lie in the interior of $\bar{B}_{2}(v(q), \| q-$ $v(q) \|)$. Consequently, $\|\mathrm{CC}(Q)-v(q)\|<\|q-v(q)\|$. Then, from $\|\mathrm{CC}(Q)-v(q)\|^{2}=$ $\|\mathrm{CC}(Q)-q\|^{2}+\|q-v(q)\|^{2}-2(q-\mathrm{CC}(Q)) \cdot(q-v(q))$, we deduce

$$
2(q-\mathrm{CC}(Q)) \cdot(q-v(q))-\|\mathrm{CC}(Q)-q\|^{2}=\|q-v(q)\|^{2}-\|\mathrm{CC}(Q)-v(q)\|^{2}>0,
$$

which implies the desired result. 
Let us now prove (iii). If $q \in \operatorname{IC}(Q)$, the claim is obviously satisfied since $\operatorname{Ln}\left[\operatorname{sm}_{Q}\right](q)=0$. Assume then that $q \notin \operatorname{IC}(Q)$. By Proposition 3.3 $0 \notin \partial \operatorname{sm}_{Q}(q)$, and hence $\operatorname{Ln}\left[\operatorname{sm}_{Q}\right](q) \neq 0$. Let us prove $\operatorname{Ln}\left[\operatorname{sm}_{Q}\right](q) \cdot n_{e}>0$ reasoning by contradiction. If $\operatorname{Ln}\left[\operatorname{sm}_{Q}\right](q) \cdot n_{e} \leq 0$, then $d / d t\left(\mathrm{D}_{e}\left(q+t \operatorname{Ln}\left[\operatorname{sm}_{Q}\right](q)\right)\right)_{t=0}=n_{e} \cdot \operatorname{Ln}\left[\operatorname{sm}_{Q}\right](q) \leq 0$, which implies that $\mathrm{D}_{e}\left(q+t \operatorname{Ln}\left[\operatorname{sm}_{Q}\right](q)\right) \leq \mathrm{D}_{e}(q)=\operatorname{sm}_{Q}(q)$ for $t>0$ small enough. On the other hand, invoking Theorem [2.6] for the function $-\operatorname{sm}_{Q}$, we have that $\operatorname{sm}_{Q}(q)+t\left\|\operatorname{Ln}\left[\operatorname{sm}_{Q}\right](q)\right\|^{2} / 2 \leq \operatorname{sm}_{Q}\left(q+t \operatorname{Ln}\left[\operatorname{sm}_{Q}\right](q)\right) \leq \mathrm{D}_{e}\left(q+t \operatorname{Ln}\left[\operatorname{sm}_{Q}\right](q)\right)$. Gathering both facts, we conclude $t\left\|\operatorname{Ln}\left[\operatorname{sm}_{Q}\right](q)\right\|^{2} / 2 \leq 0$, which is a contradiction.

Let us now prove (iv). By definition, $\mathrm{D}_{e}(q) \leq \operatorname{IR}(Q)$. This inequality is strict if $q \notin \operatorname{IC}(Q)$. Let $x \in \operatorname{IC}(Q)$. If we take a point $O$ in the edge $e$, then the function $\mathrm{D}_{e}$ can be expressed as $\mathrm{D}_{e}(p)=(p-O) \cdot n_{e}$. Then, we have

$$
\mathrm{D}_{e}(x)=(x-O) \cdot n_{e}=(x-q) \cdot n_{e}+(q-O) \cdot n_{e}=(x-q) \cdot n_{e}+\mathrm{D}_{e}(q) .
$$

Since $\mathrm{D}_{e}(x) \geq \operatorname{sm}_{Q}(x)=\operatorname{IR}(Q)$, we conclude that $(x-q) \cdot n_{e} \geq \operatorname{IR}(Q)-\mathrm{D}_{e}(q) \geq 0$, and that the second inequality is strict if $q \notin \operatorname{IC}(Q)$.

4. Analysis of the multi-center functions. Here we study the locational optimization functions $\mathcal{H}_{\mathrm{DC}}$ and $\mathcal{H}_{\mathrm{SP}}$ for the disk-covering and sphere-packing problems. We characterize their smoothness properties, generalized gradients, and critical points for arbitrary numbers of generators.

4.1. Smoothness and generalized gradients. We start by providing some alternative expressions and useful quantities. We write

$$
\mathcal{H}_{\mathrm{DC}}(P)=\max _{i \in\{1, \ldots, n\}} G_{i}(P), \quad \mathcal{H}_{\mathrm{SP}}(P)=\min _{i \in\{1, \ldots, n\}} F_{i}(P),
$$

where

$$
G_{i}(P)=\max _{q \in V_{i}(P)}\left\|q-p_{i}\right\|, \quad F_{i}(P)=\min _{q \notin \operatorname{int}\left(V_{i}(P)\right)}\left\|q-p_{i}\right\| .
$$

Note that $G_{i}(P)=\lg _{V_{i}(P)}\left(p_{i}\right)$ and $F_{i}(P)=\operatorname{sm}_{V_{i}(P)}\left(p_{i}\right)$, where, for $i \in\{1, \ldots, n\}$,

$$
\lg _{V_{i}}: V_{i} \rightarrow \mathbb{R}, \quad \operatorname{sm}_{V_{i}}: V_{i} \rightarrow \mathbb{R} .
$$

Proposition 3.3 provides an explicit expression for the generalized gradients of $\lg _{V_{i}}$ and $\operatorname{sm}_{V_{i}}$ when the Voronoi cell $V_{i}$ is held fixed. Despite the slight abuse of notation, it is convenient to let $\partial \lg _{V_{i}(P)}\left(p_{i}\right)$ denote $\partial \lg _{V}\left(p_{i}\right)_{\mid V=V_{i}(P)}$, and let $\partial \operatorname{sm}_{V_{i}(P)}\left(p_{i}\right)$ denote $\partial \operatorname{sm}_{V}\left(p_{i}\right)_{\mid V=V_{i}(P)}$.

In contrast to this analysis at fixed Voronoi partition, the properties of the functions $G_{i}$ and $F_{i}$ are strongly affected by the dependence on the Voronoi partition $\mathcal{V}(P)$. We endeavor to characterize these properties in order to study $\mathcal{H}_{\mathrm{DC}}$ and $\mathcal{H}_{\mathrm{SP}}$.

Proposition 4.1. The functions $G_{i}, F_{i}: Q^{n} \rightarrow \mathbb{R}$ are locally Lipschitz and regular. As a consequence, the locational optimization functions $\mathcal{H}_{D C}, \mathcal{H}_{S P}: Q^{n} \rightarrow \mathbb{R}$ are locally Lipschitz and regular.

Proof. (a) $G_{i}$ is locally Lipschitz and regular. The definition of the function $G_{i}$ admits the following alternative expression

$$
G_{i}(P)=\max _{v \in \operatorname{Ve}\left(V_{i}\right)}\left\|p_{i}-v\right\| .
$$


Let $P_{0}$ be nondegenerate at the $i$ th generator. Then, there exists a neighborhood $U$ of $P_{0}$ where the set $\mathcal{N}(i)$ does not change. Let $\left\{v_{1}, \ldots, v_{M_{1}}\right\},\left\{w_{1}, \ldots, w_{M_{2}}\right\}$, $\left\{z_{1}, \ldots, z_{M_{3}}\right\}$ be the vertexes of $V_{i}$ of types (a), (b) and (c) respectively. Then, $G_{i}$ can be locally written as

$$
G_{i}(P)=\max \left\{\max _{\ell \in\left\{1, \ldots, M_{1}\right\}}\left\|v_{\ell}-p_{i}\right\|, \max _{\ell \in\left\{1, \ldots, M_{2}\right\}}\left\|w_{\ell}-p_{i}\right\|, \max _{\ell \in\left\{1, \ldots, M_{3}\right\}}\left\|z_{\ell}-p_{i}\right\|\right\},
$$

for all $P \in U$. Therefore, $G_{i}$ restricted to $U$ coincides with the function $\mathcal{G}_{\mathcal{N}(i)}: Q^{n} \rightarrow$ $\mathbb{R}$ defined by

$$
\mathcal{G}_{\mathcal{N}(i)}(P)=\max \left\{\max _{\ell \in\left\{1, \ldots, M_{1}\right\}}\left\|v_{\ell}-p_{i}\right\|, \max _{\ell \in\left\{1, \ldots, M_{2}\right\}}\left\|w_{\ell}-p_{i}\right\|, \max _{\ell \in\left\{1, \ldots, M_{3}\right\}}\left\|z_{\ell}-p_{i}\right\|\right\} .
$$

The function $\mathcal{G}_{\mathcal{N}(i)}$ is the maximum of a fixed finite set of locally Lipschitz and regular functions, and consequently, locally Lipschitz and regular by Proposition 2.4 We conclude that $G_{i}$ is both locally Lipschitz and regular at $P_{0}$.

Let $P_{0}$ be degenerate at the $i$ th generator. Then, in any neighborhood $U$ of $P_{0}$ there are different sets of neighbors of the $i$ th generator. Indeed, because the number of generators, edges of the boundary $Q$ and vertexes of $Q$ is finite, there is only a finite number of different sets of neighbors of the $i$ th generator over $U$, say $\mathcal{N}^{1}(i), \ldots, \mathcal{N}^{L}(i)$. This implies that $G_{i}$ admits the following alternative expression over $U$

$$
G_{i}(P)=\min \left\{\mathcal{G}_{\mathcal{N}^{1}(i)}(P), \ldots, \mathcal{G}_{\mathcal{N}^{L}(i)}(P)\right\} .
$$

Again resorting to Proposition 2.4 we conclude that $G_{i}$ is both locally Lipschitz and regular at $P_{0}$.

(b) $F_{i}$ is locally Lipschitz and regular. From the definition of $F_{i}$, it is clear that its value at a configuration $P$ is attained at the boundary of the Voronoi region $V_{i}$. Therefore, one only minimizes among the edges associated with the Voronoi neighbors $\mathcal{N}(i)$ and the edges of $Q$ with non-empty intersection with $V_{i}$. Moreover, one can also see that the minimum must be attained at a point of the form $\operatorname{proj}_{e}\left(p_{i}\right)$, for some edge $e$ of $V_{i}$. Now, consider the function $\mathcal{F}_{\mathcal{N}(i)}: Q^{n} \rightarrow \mathbb{R}$ defined by

$$
\mathcal{F}_{\mathcal{N}(i)}(P)=\min \left\{\min _{j \in\{1, \ldots, n\}}\left\|p_{i}-\frac{p_{i}+p_{j}}{2}\right\|, \min _{e \in \operatorname{Ed}(Q)} \mathrm{D}_{e}\left(p_{i}\right)\right\}
$$

We shall prove that $\mathcal{F}_{\mathcal{N}(i)}$ coincides with $F_{i}$. If $k \notin \mathcal{N}(i)$, then $\left(p_{i}+p_{k}\right) / 2 \notin V_{i}$. Since $Q \backslash V_{i}$ is open, there exists a neighborhood of $\left(p_{i}+p_{k}\right) / 2$ such that $U \subset Q / V_{i}$. Therefore,

$$
\left\|p_{i}-\frac{p_{i}+p_{j}}{2}\right\|>\min _{q \in U}\left\|p_{i}-q\right\| \geq \min _{q \notin \operatorname{int}\left(V_{i}\right)}\left\|p_{i}-q\right\|=F_{i}(P) .
$$

If an edge $e$ of $Q$ does not intersect $V_{i}$, then $\operatorname{proj}_{e}\left(p_{i}\right) \notin V_{i}$. Using again the fact that $Q \backslash V_{i}$ is open, there exists a neighborhood $U$ of $\operatorname{proj}_{e}\left(p_{i}\right)$ in $\mathbb{R}^{2}$ such that $U \cap Q \subset Q \backslash V_{i}$. Then,

$$
\left\|p_{i}-\operatorname{proj}_{e}\left(p_{i}\right)\right\|>\min _{q \in U \cap Q}\left\|p_{i}-q\right\| \geq F_{i}(P) .
$$

As a consequence of the previous inequalities, $F_{i}$ equals $\mathcal{F}_{\mathcal{N}(i)}$. Being the minimum of a fixed finite number of locally Lipschitz and regular functions on $Q^{n}, F_{i}$ is also locally Lipschitz and regular by Proposition 2.4 
Next, one can actually prove the following stronger result.

Proposition 4.2. The locational optimization functions $\mathcal{H}_{D C}, \mathcal{H}_{S P}: Q^{n} \rightarrow \mathbb{R}$ are globally Lipschitz, with Lipschitz constant equal to 1.

Proof. (a) $\mathcal{H}_{D C}$ is globally Lipschitz. Let $P, P^{\prime}$ be two configurations of the $n$ generators. Without loss of generality, assume that $\mathcal{H}_{\mathrm{DC}}(P) \leq \mathcal{H}_{\mathrm{DC}}\left(P^{\prime}\right)$. Let $i, j$ and $q_{0}, q_{0}^{\prime} \in Q$ be such that $\mathcal{H}_{\mathrm{DC}}(P)=G_{i}(P)=\left\|q_{0}-p_{i}\right\|$ and $\mathcal{H}_{\mathrm{DC}}\left(P^{\prime}\right)=G_{j}\left(P^{\prime}\right)=$ $\left\|q_{0}^{\prime}-p_{j}^{\prime}\right\|$. Now, consider the set $B_{2}\left(q_{0}^{\prime}, G_{i}(P)\right)$. Then there exists a $k$ such that $p_{k} \in \bar{B}_{2}\left(q_{0}^{\prime}, G_{i}(P)\right)$ (otherwise, $\left\|q_{0}^{\prime}-p_{l}\right\|>G_{i}(P)$, which contradicts the definition of the function $\left.\mathcal{H}_{\mathrm{DC}}\right)$. On the other hand, we necessarily have that $p_{k}^{\prime} \notin B_{2}\left(q_{0}^{\prime}, G_{j}\left(P^{\prime}\right)\right)$, since otherwise $\left\|q_{0}^{\prime}-p_{k}^{\prime}\right\|<\left\|q_{0}^{\prime}-p_{j}^{\prime}\right\|$, which implies that $q_{0}^{\prime} \notin V_{j}^{\prime}$, contradiction. Finally, we apply the triangle inequality to obtain $\left\|q_{0}^{\prime}-p_{k}^{\prime}\right\| \leq\left\|q_{0}^{\prime}-p_{k}\right\|+\left\|p_{k}-p_{k}^{\prime}\right\|$. Gathering the previous facts, we have

$$
\begin{aligned}
\left|\mathcal{H}_{\mathrm{DC}}\left(P^{\prime}\right)-\mathcal{H}_{\mathrm{DC}}(P)\right|=G_{j}\left(P^{\prime}\right)-G_{i}(P) & \\
& \leq\left\|q_{0}^{\prime}-p_{k}^{\prime}\right\|-\left\|q_{0}^{\prime}-p_{k}\right\| \leq\left\|p_{k}-p_{k}^{\prime}\right\| \leq\left\|P-P^{\prime}\right\| .
\end{aligned}
$$

(b) $\mathcal{H}_{S P}$ is globally Lipschitz. Let $P, P^{\prime}$ be two configurations of the $n$ generators. Without loss of generality, assume that $\mathcal{H}_{\mathrm{SP}}(P) \leq \mathcal{H}_{\mathrm{SP}}\left(P^{\prime}\right)$. Let $i, j$ and $q_{0}, q_{0}^{\prime} \in Q$ be such that $\mathcal{H}_{\mathrm{SP}}(P)=F_{i}(P)=\left\|q_{0}-p_{i}\right\|$ and $\mathcal{H}_{\mathrm{SP}}\left(P^{\prime}\right)=F_{j}\left(P^{\prime}\right)=\left\|q_{0}^{\prime}-p_{j}^{\prime}\right\|$. We treat separately the following two cases: (i) $q_{0}$ does not belong to the boundary of $Q$, and (ii) $q_{0}$ belongs to the boundary of $Q$. In case (i), it necessarily exists $k \in \mathcal{N}(i)$ such that $\left\|q_{0}-p_{i}\right\|=\left\|q_{0}-p_{k}\right\|$. If $\left\|q_{0}-p_{i}^{\prime}\right\| \geq F_{j}\left(P^{\prime}\right)$, then

$$
\begin{aligned}
\left|\mathcal{H}_{\mathrm{SP}}\left(P^{\prime}\right)-\mathcal{H}_{\mathrm{SP}}(P)\right|=F_{j}\left(P^{\prime}\right)-F_{i}(P) & \leq\left\|q_{0}-p_{i}^{\prime}\right\|-\left\|q_{0}-p_{i}\right\| \\
& \leq\left\|p_{i}-p_{i}^{\prime}\right\| \leq\left\|P-P^{\prime}\right\| .
\end{aligned}
$$

If, on the contrary, $\left\|q_{0}-p_{i}^{\prime}\right\|<F_{j}\left(P^{\prime}\right)$, then $q_{0} \in \operatorname{int}\left(V_{i}^{\prime}\right)$. Therefore, $\left\|q_{0}-p_{k}^{\prime}\right\| \geq$ $F_{k}\left(P^{\prime}\right) \geq F_{j}\left(P^{\prime}\right)$. Now, we perform the same computation as in 4.5) to conclude $\left|\mathcal{H}_{\mathrm{SP}}\left(P^{\prime}\right)-\mathcal{H}_{\mathrm{SP}}(P)\right| \leq\left\|P-P^{\prime}\right\|$.

In case (ii), we prove that $\left\|q_{0}-p_{i}^{\prime}\right\| \geq F_{j}\left(P^{\prime}\right)$. Suppose this is not true, i.e., $\left\|q_{0}-p_{i}^{\prime}\right\|<F_{j}\left(P^{\prime}\right)$. Let $m=q_{0}+\epsilon\left(q_{0}-p_{i}^{\prime}\right)$, with sufficiently small $\epsilon>0$ such that $\left\|m-p_{i}^{\prime}\right\|<F_{j}\left(P^{\prime}\right)$. Clearly $m \notin Q$. On the other hand, by definition $B_{2}\left(p_{i}^{\prime}, F_{i}\left(P^{\prime}\right)\right) \subset$ $V_{i}^{\prime}$. Now, we have,

$$
B_{2}\left(p_{i}^{\prime}, F_{j}\left(P^{\prime}\right)\right) \subset B_{2}\left(p_{i}^{\prime}, F_{i}\left(P^{\prime}\right)\right) \subset V_{i}^{\prime} \subset Q .
$$

But, since $\left\|m-p_{i}^{\prime}\right\|<F_{j}\left(P^{\prime}\right)$, then $m \in B_{2}\left(p_{i}^{\prime}, F_{j}\left(P^{\prime}\right)\right) \subset Q$, which is a contradiction. Therefore, $\left\|q_{0}-p_{i}^{\prime}\right\| \geq F_{j}\left(P^{\prime}\right)$, and now the same argument as in 44.5) guarantees that $\left|\mathcal{H}_{\mathrm{SP}}\left(P^{\prime}\right)-\mathcal{H}_{\mathrm{SP}}(P)\right| \leq\left\|P-P^{\prime}\right\|$.

We now introduce some quantities that are useful in characterizing the generalized gradient of the functions $G_{i}$. Given a vertex of type (b), $v=v(e, i, j)$, determined by the edge $e$ and two generators $p_{i}$ and $p_{j}$, we consider the scalar function $\lambda(e, i, j)$ defined by

$$
\operatorname{proj}_{e}\left(p_{j}-v(e, i, j)\right)=\lambda(e, i, j) \operatorname{proj}_{e}\left(p_{j}-p_{i}\right)
$$

where $P_{e}$ is the orthogonal projection onto the edge $e$; see Fig. 4.1 One can see that $\lambda(e, i, j)+\lambda(e, j, i)=1$. If $e$ is a segment in the line $a x+b y+c=0,\left(\Delta x_{i j}, \Delta y_{i j}\right)=$ $p_{j}-p_{i},\left(x_{m}, y_{m}\right)=\left(p_{i}+p_{j}\right) / 2$, then one can show

$$
\lambda(e, i, j)=\frac{1}{2}-\frac{\left(a \Delta x_{i j}+b \Delta y_{i j}\right)\left(a x_{m}+b y_{m}+c\right)}{\left(a \Delta y_{i j}-b \Delta x_{i j}\right)^{2}} .
$$



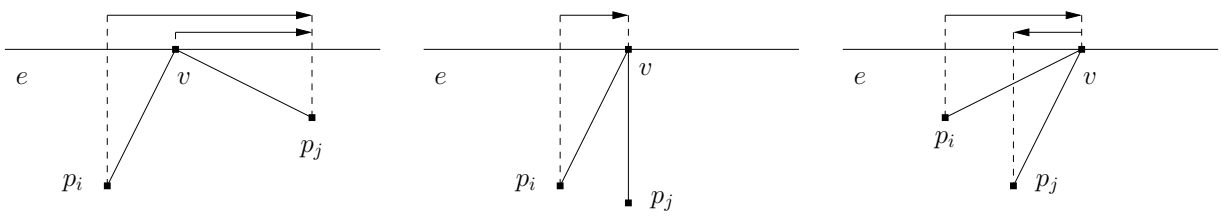

FIG. 4.1. To illustrate eq. 4.6 we draw the vectors $\operatorname{proj}_{e}\left(p_{j}-v(e, i, j)\right)$ and $\operatorname{proj}_{e}\left(p_{j}-p_{i}\right)$ for various locations of $p_{i}, p_{j}$, and $e$. The left, center and right figures correspond to $\lambda(e, i, j)>0$, $\lambda(e, i, j)=0, \lambda(e, i, j)<0$, respectively.

Given a vertex of type (a), $v=v(i, j, k)$, determined by the three generators $p_{i}, p_{j}$, and $p_{k}$, we consider the scalar function $\mu(i, j, k)$ defined by

$$
\operatorname{proj}_{e_{j k}}\left(p_{\ell}-v(i, j, k)\right)=\mu(i, j, k) \operatorname{proj}_{e_{j k}}\left(p_{\ell}-p_{i}\right)
$$

where $e_{j k}$ is the bisector of $p_{j}$ and $p_{k}$ and where $p_{\ell}=p_{j}$ if $p_{j}$ belongs to the half-plane defined by $e_{j k}$ containing $p_{i}$, and $p_{\ell}=p_{k}$ otherwise. One can see that $\mu(i, j, k)=$ $\mu(i, k, j)$ and that $\mu(i, j, k)+\mu(j, k, i)+\mu(k, i, j)=1$. From the expression for $\lambda$, one can obtain

$$
\mu(i, j, k)=\frac{1}{2}+\frac{\left(\Delta x_{i j} \Delta x_{j k}+\Delta y_{i j} \Delta y_{j k}\right)\left(\Delta x_{i k} \Delta x_{j k}+\Delta y_{i k} \Delta y_{j k}\right)}{2\left(x_{k} \Delta y_{i j}-x_{j} \Delta y_{i k}+x_{i} \Delta y_{j k}\right)^{2}} .
$$

Note that, in general, $\lambda$ and $\mu$ are not positive functions. Now we are ready to describe in detail the structure of the generalized gradient of the functions $G_{i}, F_{i}$.

Proposition 4.3. The generalized gradient of $G_{i}: Q^{n} \rightarrow \mathbb{R}$ at $P \in Q^{n}$ is

$$
\partial G_{i}(P)=\operatorname{co}\left\{\partial_{v} G_{i}(P) \in\left(\mathbb{R}^{2}\right)^{n} \mid v \in \operatorname{Ve}\left(V_{i}(P)\right) \text { such that } G_{i}(P)=\left\|p_{i}-v\right\|\right\}
$$

where we consider separately the following cases. If $v=v(i, j, k)$ is a nondegenerate vertex of type (a), then

$$
\begin{aligned}
& \partial_{v(i, j, k)} G_{i}(P)=\partial_{v(k, i, j)} G_{k}(P)=\partial_{v(j, k, i)} G_{j}(P)= \\
& (0, \ldots, \underbrace{\mu(i, j, k) \operatorname{vrs}\left(p_{i}-v\right)}_{\text {ith place }}, \ldots, \underbrace{\mu(j, k, i) \operatorname{vrs}\left(p_{j}-v\right)}_{j \text { th place }}, \ldots, \underbrace{\mu(k, i, j) \operatorname{vrs}\left(p_{k}-v\right)}_{\text {kth place }}, \ldots, 0)
\end{aligned}
$$

where, without loss of generality, we let $i<j<k$. If $v=v(e, i, j)$ is a nondegenerate vertex of type (b), then

$$
\begin{aligned}
\partial_{v(e, i, j)} G_{i}(P)=\partial_{v(e, j, i)} G_{j}(P) & =(0, \ldots, \underbrace{\lambda(e, i, j) \operatorname{vrs}\left(p_{i}-v\right)}_{\text {ith place }}, \ldots, \underbrace{\lambda(e, j, i) \operatorname{vrs}\left(p_{j}-v\right)}_{j \text { th place }}, \ldots, 0)
\end{aligned}
$$

where, without loss of generality, we let $i<j$. If $v=v(e, f, i)$ is a nondegenerate vertex of type (c), then

$$
\partial_{v(e, f, i)} G_{i}(P)=(0, \ldots, 0, \underbrace{\operatorname{vrs}\left(p_{i}-v\right)}_{\text {ith place }}, 0, \ldots, 0) .
$$

Finally, if the vertex $v$ is degenerate, i.e., if $v$ is determined by $d>3$ elements (generators or edges), then there are $\left(\begin{array}{c}d-1 \\ 2\end{array}\right)$ pairs of elements which determine the 
vertex $v$ together with the generator $p_{i}$. In this case, $\partial_{v} G_{i}(P)$ is the convex hull of $\partial_{v(\alpha, \beta, \gamma)} G_{i}(P)$ for all $\left(\begin{array}{c}d-1 \\ 2\end{array}\right)$ such triplets $(\alpha, \beta, \gamma)$.

Note that, at all nondegenerate configurations $P$, the quantity $\partial_{v} G_{i}(P)$ is the generalized gradient of the function $\left(p_{1}, \ldots, p_{n}\right) \mapsto\left\|p_{i}-v(i, j, k)\right\|$; however, this interpretation cannot be given when $P$ is degenerate.

Proof. We present the proof for the expression for $\partial G_{i}(P)$. Let us consider first the case when $P$ is nondegenerate configuration for the $i$ th generator. According to the proof of Proposition $4.1 G_{i}$ coincides with the function $\mathcal{G}_{\mathcal{N}(i)}$ over a neighborhood $U$ of $P$. Hence, $\partial G_{i}(P)=\partial \mathcal{G}_{\mathcal{N}(i)}(P)$ which, according to eq. (4.2) and Proposition 2.4 takes the form

$$
\operatorname{co}\left\{\frac{\partial}{\partial P}\left\|v-p_{i}\right\| \mid v \in \operatorname{Ve}\left(V_{i}(P)\right) \text { such that }\left\|v-p_{i}\right\|=G_{i}(P)\right\} .
$$

If $v=v(i, j, k)$ is a nondegenerate vertex of type (a), then

$$
\begin{aligned}
& \frac{\partial}{\partial p_{i}}\left\|p_{i}-v(i, j, k)\right\|=\operatorname{vrs}\left(p_{i}-v\right)\left(I_{2}-\frac{\partial v}{\partial p_{i}}\right)=\mu(i, j, k) \operatorname{vrs}\left(p_{i}-v\right), \\
& \frac{\partial}{\partial p_{j}}\left\|p_{i}-v(i, j, k)\right\|=-\operatorname{vrs}\left(p_{i}-v\right)\left(\frac{\partial v}{\partial p_{j}}\right)=\mu(j, k, i) \operatorname{vrs}\left(p_{j}-v\right), \\
& \frac{\partial}{\partial p_{\ell}}\left\|p_{i}-v(i, j, k)\right\|=0, \quad \ell \neq i, j, k .
\end{aligned}
$$

If $v=v(e, i, j)$ is a nondegenerate vertex of type (b), then

$$
\begin{aligned}
& \frac{\partial}{\partial p_{i}}\left\|p_{i}-v(e, i, j)\right\|=\operatorname{vrs}\left(p_{i}-v\right)\left(I_{2}-\frac{\partial v}{\partial p_{i}}\right)=\lambda(e, i, j) \operatorname{vrs}\left(p_{i}-v\right), \\
& \frac{\partial}{\partial p_{j}}\left\|p_{i}-v(e, i, j)\right\|=-\operatorname{vrs}\left(p_{i}-v\right)\left(\frac{\partial v}{\partial p_{j}}\right)=\lambda(e, j, i) \operatorname{vrs}\left(p_{j}-v\right), \\
& \frac{\partial}{\partial p_{\ell}}\left\|p_{i}-v(e, i, j)\right\|=0, \quad \ell \neq i, j .
\end{aligned}
$$

If $v=v(e, f, i)$ is a nondegenerate vertex of type (c), then

$$
\begin{aligned}
& \frac{\partial}{\partial p_{i}}\left\|p_{i}-v(e, f, i)\right\|=\operatorname{vrs}\left(p_{i}-v\right), \\
& \frac{\partial}{\partial p_{\ell}}\left\|p_{i}-v(e, f, i)\right\|=0, \quad \ell \neq i .
\end{aligned}
$$

If $P$ is a degenerate configuration at the $i$ th generator, then this function can be expressed as in eq. (4.3) in a sufficiently small neighborhood $U$ of $P$. According to Proposition [2.4 the generalized gradient of $G_{i}$ is given by the convex hull of the generalized gradients of each of the functions $\mathcal{G}_{\mathcal{N}^{1}(i)}, \ldots, \mathcal{G}_{\mathcal{N}^{L}(i)}$. The claim now follows by reproducing the previous discussion for the generalized gradients of each of the functions $\mathcal{G}_{\mathcal{N}^{\ell}(i)}, \ell \in\{1, \ldots, L\}$.

The expression for $\partial F_{i}(P)$ can be deduced in an analogous (and simpler) way, since according to the proof of Proposition 4.1 it is not necessary to establish any distinction between the degenerate and the nondegenerate configurations. Accordingly, we state the following result without proof.

Proposition 4.4. The generalized gradient of $F_{i}: Q^{n} \rightarrow \mathbb{R}$ at $P \in Q^{n}$ is

$$
\partial F_{i}(P)=\operatorname{co}\left\{\partial_{e} F_{i}(P) \in\left(\mathbb{R}^{2}\right)^{n} \mid e \in \operatorname{Ed}\left(V_{i}(P)\right) \text { such that } F_{i}(P)=\mathrm{D}_{e}\left(p_{i}\right)\right\}
$$


where, if $e=e(i, j)$ is an edge of type (a), then

$$
\partial_{e(i, j)} F_{i}(P)=\partial_{e(j, i)} F_{j}(P)=\frac{1}{2}(0, \ldots, \underbrace{n_{e(i, j)}}_{\text {ith place }}, \ldots, \underbrace{-n_{e(i, j)}}_{j \text { th place }}, \ldots, 0),
$$

and if $e=e(i)$ is an edge of type (b), then

$$
\partial_{e(i)} F_{i}(P)=(0, \ldots, \underbrace{n_{e(i)}}_{\text {ith place }}, \ldots, 0) .
$$

Next, we give conditions under which the functions $\lambda$ and $\mu$ take positive values. Lemma 4.5. Let $P \in Q^{n}$ and let $v \in \operatorname{Ve}_{\mathrm{DC}}(\mathcal{V}(P))$. Then,

(i) if $v$ belongs to an edge e of $Q$, then there exist generators $p_{i}$ and $p_{j}$ such that $\lambda(e, i, j)$ and $\lambda(e, j, i)$ are positive, and

(ii) if $v$ belongs to $\operatorname{int}(Q)$, then there exist generators $p_{i}, p_{j}$ and $p_{k}$ such that $\mu(i, j, k), \mu(j, k, i)$ and $\mu(k, i, j)$ are positive.

Proof. Consider first the case when $v$ is nondegenerate. If $v$ is in the edge $e$ of $Q$ (i.e. $v$ is of type (b)), let $p_{i}$ and $p_{j}$ the two generators determining it. From the definition of $\lambda$, one sees that the values $\lambda(e, i, j)=0$ and $\lambda(e, j, i)=0$ correspond to, respectively, $p_{j}$ and $p_{i}$ lying on the orthogonal line to $e$ passing through $v(e, i, j)$. If $\lambda(e, i, j) \leq 0$, then there exists $w \in e \cap V_{j}$ such that $\left\|p_{j}-w\right\|>\left\|p_{j}-v\right\|=\mathcal{H}_{\mathrm{DC}}(P)$, which is a contradiction. Therefore $\lambda(e, i, j)>0$. The same argument guarantees $\lambda(e, j, i)>0$. If $v$ is of type (a), and $p_{i}, p_{j}$ and $p_{k}$ are the elements determining it, a similar argument leads to the conclusion that $\mu(i, j, k), \mu(j, k, i)$ and $\mu(k, i, j)$ are positive.

Consider the case when $v$ is degenerate. Let $\left\{i_{1}, \ldots, i_{m}\right\}$ be such that $v \in V_{i_{j}}$, $j \in\{1, \ldots, m\}$. Assume $v$ is an edge $e$ of $Q$. Let $l$ denote the orthogonal line to the edge $e$ passing through $v$. We claim that there must exist generators in $\left\{i_{1}, \ldots, i_{m}\right\}$ on both sides of $l$. Assume this is not the case, i.e., $\left\{p_{i_{1}}, \ldots, p_{i_{m}}\right\}$ are contained in one of the closed half-planes defined by $l$, say $l_{-}$. Take $w \in l_{+} \cap e$ arbitrarily close to $v$. Since $\left\{p_{i_{1}}, \ldots, p_{i_{m}}\right\} \subset l_{-}$, we have $\left\|p_{i_{j}}-w\right\|>\left\|p_{i_{j}}-v\right\|$. On the other hand, since no generator outside the set $\left\{p_{i_{1}}, \ldots, p_{i_{m}}\right\}$ is involved in the definition of $v$, there must exist $j^{*}$ such that $w \in V_{i_{j^{*}}}$. Therefore, $G_{i_{j^{*}}}(P) \geq\left\|p_{i_{j^{*}}}-w\right\|>\left\|p_{i_{j^{*}}}-v\right\|=\mathcal{H}_{\mathrm{DC}}(P)$, which is a contradiction. Assume now that $v \in \operatorname{int}(Q)$. Our claim is that, for any line $l$ passing through $v$, there must exist generators on both sides of $l$. If this is not the case, i.e., $\left\{p_{i_{1}}, \ldots, p_{i_{m}}\right\} \subset l_{-}$, then take $w \in B_{2}(v, \epsilon) \cap l_{+} \cap o$, where $o$ denotes the orthogonal line to $l$ passing through $v$. As before, $w \in V_{i_{j^{*}}}$ for some $j^{*}$ and $\left\|p_{i_{j^{*}}}-w\right\|>\left\|p_{i_{j^{*}}}-v\right\|$, which yields a contradiction.

This completes our analysis of the generalized gradients of $G_{i}$ and $F_{i}$ and, with these results, we return to studying the generalized gradients of $\mathcal{H}_{\mathrm{DC}}$ and $\mathcal{H}_{\mathrm{SP}}$. An immediate consequence of Propositions [2.4 and 4.1] is that

$$
\begin{aligned}
\partial \mathcal{H}_{\mathrm{DC}}(P) & =\operatorname{co}\left\{\partial G_{i}(P) \mid i \in I(P)\right\}, \\
\partial \mathcal{H}_{\mathrm{SP}}(P) & =\operatorname{co}\left\{\partial F_{i}(P) \mid i \in I(P)\right\} .
\end{aligned}
$$

Furthermore we can provide the following more detailed characterization.

Proposition 4.6. Let $P \in Q^{n}$. For each $i \in\{1, \ldots, n\}$, the image by $\pi_{i}$ of the 
generalized gradients of $\mathcal{H}_{D C}$ and $\mathcal{H}_{S P}$ at $P$ is given by

$$
\begin{gathered}
\pi_{i}\left(\partial \mathcal{H}_{D C}(P)\right)= \begin{cases}\pi_{i}\left(\partial G_{i}(P)\right) & \text { if } i \in I(P), \operatorname{Ve} \mathrm{DC}(\mathcal{V}(P)) \subset \operatorname{Ve}\left(V_{i}(P)\right) \\
\operatorname{co}\left\{\pi_{i}\left(\partial G_{i}(P)\right), 0\right\} & \text { if } i \in I(P), \operatorname{Ve} \mathrm{D}_{\mathrm{DC}}(\mathcal{V}(P)) \not \subset \operatorname{Ve}\left(V_{i}(P)\right) \\
0 & \text { if } i \notin I(P)\end{cases} \\
\pi_{i}\left(\partial \mathcal{H}_{S P}(P)\right)= \begin{cases}\pi_{i}\left(\partial F_{i}(P)\right) & \text { if } i \in I(P), \operatorname{Ed} \mathrm{SP}(\mathcal{V}(P)) \subset \operatorname{Ed}\left(V_{i}(P)\right) \\
\operatorname{co}\left\{\pi_{i}\left(\partial F_{i}(P)\right), 0\right\} & \text { if } i \in I(P), \operatorname{Ed} \mathrm{SP}(\mathcal{V}(P)) \not \subset \operatorname{Ed}\left(V_{i}(P)\right) \\
0 & \text { if } i \notin I(P)\end{cases}
\end{gathered}
$$

Proof. From eq. 4.7), if $i \notin I(P)$, then $\pi_{i}\left(\partial \mathcal{H}_{\mathrm{DC}}(P)\right)=0, \pi_{i}\left(\partial \mathcal{H}_{\mathrm{SP}}(P)\right)=0$. If $i \in I(P)$, then using Proposition 4.3 we deduce that the generators $p_{j}$ such that $\partial G_{j}$ has a nonzero entry in the $i$ th place (and hence contributes to the projection by $\pi_{i}$ of $\left.\partial \mathcal{H}_{\mathrm{DC}}\right)$ must share a vertex with the $i$ th generator. Analogously, if $i \in I(P)$, then using Proposition 4.4 we deduce that the generators $p_{j}$ such that $\partial F_{j}$ has a nonzero entry in the $i$ th place (and hence contributes to the projection by $\pi_{i}$ of $\partial \mathcal{H}_{\mathrm{SP}}$ ) must satisfy $j \in \mathcal{N}(i)$. For the disk-covering function, if $v$ is a common vertex of $V_{i}$ and $V_{j}$, determined by $i, j$ and a third element $\alpha$, then $\partial_{v(\alpha, j, i)} G_{j}=\partial_{v(\alpha, i, j)} G_{i}$, and the expression for $\pi_{i}\left(\partial \mathcal{H}_{\mathrm{DC}}(P)\right)$ then follows. The argument for the expression of $\pi_{i}\left(\partial \mathcal{H}_{\mathrm{SP}}(P)\right)$ is analogous.

4.2. Critical points. Having characterized the generalized gradients of $\mathcal{H}_{\mathrm{DC}}$ and $\mathcal{H}_{\mathrm{SP}}$, we now turn to studying their critical points.

TheOrem 4.7 (Minima of $\mathcal{H}_{\mathrm{DC}}$ ). Let $P \in Q^{n}$ be a nondegenerate configuration and $0 \in \operatorname{int}\left(\partial \mathcal{H}_{D C}(P)\right)$. Then, $P$ is a strict local minimum of $\mathcal{H}_{D C}$, all generators are active and $P$ is a circumcenter Voronoi configuration.

Proof. Since $P$ is nondegenerate, note from Proposition 4.3 that $\partial_{v} G_{i}$ is a singleton for each $v \in \operatorname{Ve}\left(V_{i}(P)\right), i \in\{1, \ldots, n\}$. Let $w \in\left(\mathbb{R}^{2}\right)^{n}$. We claim that moving the configuration of the generators from $P$ in the direction $w$ can only increase the cost. The hypothesis $0 \in \operatorname{int}\left(\partial \mathcal{H}_{\mathrm{DC}}(P)\right)$ implies by Lemma 2.1 that there exists $i$ and $v \in \operatorname{Ve}\left(V_{i}(P)\right) \cap \operatorname{Ve}_{\mathrm{DC}}(\mathcal{V}(P))$ such that $w \cdot \partial_{v} G_{i}(P)>0$. Since $P$ is nondegenerate, $v$ will still belong to $V_{i}(P+\epsilon w)$ for sufficiently small $\epsilon>0$, and consequently $\mathcal{H}_{\mathrm{DC}}(P+\epsilon w) \geq G_{i}(P+\epsilon w)>G_{i}(P)=\mathcal{H}_{\mathrm{DC}}(P)$. Therefore $P$ is a strict local minimum.

Since $\pi_{i}$ is an open map, the set $\pi_{i}\left(\operatorname{int}\left(\partial \mathcal{H}_{\mathrm{DC}}(P)\right)\right)$ is open for each $i \in\{1, \ldots, n\}$. Therefore, $\pi_{i}\left(\operatorname{int}\left(\partial \mathcal{H}_{\mathrm{DC}}(P)\right)\right) \neq 0$, and hence all generators are active, i.e. $I(P)=$ $\{1, \ldots, n\}$. Let us see that all generators must also be centered. Assume $P$ is nondegenerate and consider the $i$ th generator. Take $w \in \mathbb{R}^{2}$ and let $\bar{w} \in\left(\mathbb{R}^{2}\right)^{n}$ be the vector with has $w$ in the $i$ th place and 0 otherwise. By Lemma 2.1] there exist $j$ and $v \in \operatorname{Ve}\left(V_{j}(P)\right) \cap \operatorname{Ve}_{\mathrm{DC}}(\mathcal{V}(P))$ such that $\bar{w} \cdot \partial_{v} G_{j}>0$. Since $\bar{w} \cdot \partial_{v} G_{j}=w \cdot \pi_{i}\left(\partial_{v} G_{j}\right)>0$, then necessarily $\pi_{i}\left(\partial_{v} G_{j}\right) \neq 0$, and therefore $v \in V_{i}(P)$ and $\pi_{i}\left(\partial_{v} G_{j}\right)=\pi_{i}\left(\partial_{v} G_{i}\right)$. The vertex $v$ is determined by $p_{i}, p_{j}$ and a third element, say $\alpha$. Depending on whether $\alpha$ corresponds to an edge or to another generator, we have that $\pi_{i}\left(\partial_{v} G_{i}\right)$ is equal to $\lambda(\alpha, i, j) \operatorname{vrs}\left(p_{i}-v\right)$ or $\mu(\alpha, i, j) \operatorname{vrs}\left(p_{i}-v\right)$. In any case, from Lemma 4.5 we deduce that $\lambda(\alpha, i, j)$ (respectively $\mu(\alpha, i, j))$ belongs to $(0,1)$. Therefore $w \cdot \pi_{i}\left(\partial_{v} G_{i}\right)>0$ implies $w \cdot \operatorname{vrs}\left(p_{i}-v\right)>0$. Consequently, $0 \in \operatorname{int}\left(\partial \lg _{V_{i}(P)}\left(p_{i}\right)\right)$. By Proposition 3.3 this implies that $p_{i}=\mathrm{CC}\left(V_{i}\right)$. Hence, $P$ is a circumcenter Voronoi configuration.

Theorem 4.8 (Maxima of $\mathcal{H}_{\mathrm{SP}}$ ). Let $P \in Q^{n}$ and $0 \in \operatorname{int}\left(\partial \mathcal{H}_{S P}(P)\right)$. Then, $P$ is a strict local maximum of $\mathcal{H}_{S P}$, all generators are active and $P$ is a generic incenter Voronoi configuration. 

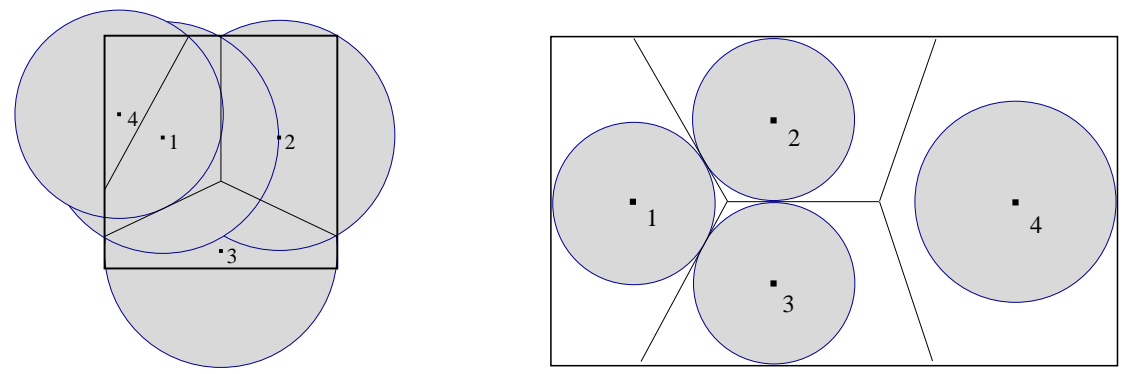

FIG. 4.2. Local extrema of the disk-covering and the sphere-packing functions in a convex polygonal environment. The configuration on the left corresponds to a local minimum of $\mathcal{H}_{D C}$ with $0 \in \partial \mathcal{H}_{D C}(P)$ and $\operatorname{int}\left(\partial \mathcal{H}_{D C}(P)\right)=\emptyset$. The configuration on the right corresponds to a local maximum of $\mathcal{H}_{S P}$ with $0 \in \partial \mathcal{H}_{S P}(P)$ and $\operatorname{int}\left(\partial \mathcal{H}_{S P}(P)\right)=\emptyset$. In both configurations, the 4 th generator is inactive and non-centered.

Proof. The proof of this result is analogous to the proof of Theorem 4.7 Note that $0 \in \operatorname{int}\left(\partial \operatorname{sm}_{V_{i}(P)}\left(p_{i}\right)\right)$ implies, by Proposition 3.3 that $\operatorname{IC}\left(V_{i}(P)\right)=\left\{p_{i}\right\}$, and hence $P$ is a generic incenter Voronoi configuration.

REMARK 4.9. Theorems 4.7 and 4.8 precisely provide the interpretation of the multicenter problems that we gave in Section 2.2 since all generators are active, they share the same radius. If one drops the hypothesis that 0 belongs to the generalized gradient of the locational optimization function, then one can think of simple examples where $P$ is a local minimum of $\mathcal{H}_{\mathrm{DC}}$ (respectively local maximum of $\mathcal{H}_{\mathrm{SP}}$ ), and there are generators which are inactive and non-centered, see Fig. 4.2

5. Dynamical systems for the multi-center problems. In this section, we describe three algorithms that (locally) extremize the multi-center functions for the disk-covering and the sphere-packing problems. We first examine the gradient flow descent associated with the locational optimization functions $\mathcal{H}_{\mathrm{DC}}$ and $\mathcal{H}_{\mathrm{SP}}$. This flow is guaranteed to find a local critical point, but it has the drawback of being centralized, as we describe later. Then, we propose two decentralized flows for each problem. One roughly consists of a distributed implementation of the gradient descent. As we show, it is very much in the spirit of behavior-based robotics. The other one follows the logical strategy given the result in Theorems 4.7 and 4.8 each generator moves toward the circumcenter (alternatively, incenter set) of its own Voronoi polygon. We call them Lloyd flows, since they resemble the original Lloyd algorithm for vector quantization problems, where each quantizer moves toward the centroid or center of mass of its own Voronoi region, see [14, 16, 21]. We present continuous-time versions of the algorithms and discuss their convergence properties. In our setting, the generators' location obeys a first order dynamical behavior described by

$$
\dot{p}_{i}=u_{i}\left(p_{1}, \ldots, p_{n}\right), \quad i \in\{1, \ldots, n\} .
$$

The dynamical system (5.1) is said to be (strongly) centralized if there exists at least an $i \in\{1, \ldots, n\}$ such that $u_{i}\left(p_{1}, \ldots, p_{n}\right)$ cannot be written as a function of the form $u_{i}\left(p_{i}, p_{i_{1}}, \ldots, p_{i_{m}}\right)$, with $m<n-1$. The dynamical system (5.1) is said to be Voronoi-distributed if each $u_{i}\left(p_{1}, \ldots, p_{n}\right)$ can be written as a function of the form $u_{i}\left(p_{i}, p_{i_{1}}, \ldots, p_{i_{m}}\right)$, with $i_{k} \in \mathcal{N}(P, i), k \in\{1, \ldots, m\}$. Finally, the dynamical system (5.1) is said to be nearest-neighbor-distributed if each $u_{i}\left(p_{1}, \ldots, p_{n}\right)$ can be written as a function of the form $u_{i}\left(p_{i}, p_{i_{1}}, \ldots, p_{i_{m}}\right)$, with $\left\|p_{i}-p_{i_{k}}\right\| \leq\left\|p_{i}-p_{j}\right\|$ for all 
$j \in\{1, \ldots, n\}$, and $k \in\{1, \ldots, m\}$. A nearest-neighbor-distributed dynamical system is also Voronoi-distributed.

It is well known that there are at most $3 n-6$ neighborhood relationships in a planar Voronoi diagram [23, see Section 2.3]. Therefore, the number of Voronoi neighbors of each site is on average less than or equal to 6. (Recall that sites are Voronoi-neighbors if they share an edge, not just a vertex.) We refer to [10] for more details on the distributed character of Voronoi neighborhood relationships.

Note that the set of indexes $\left\{i_{1}, \ldots, i_{m}\right\}$ for an specific generator $p_{i}$ of a Voronoidistributed or a nearest-neighbor-distributed dynamical system is not the same for all possible configurations $P$. In other words, the identity of both the Voronoi neighbors and the nearest neighbors might change along the evolution, i.e., the topology of the dynamical system is dynamic.

5.1. Nonsmooth gradient dynamical systems. Consider the (signed) generalized gradient descent flow (2.7) for the locational optimization functions $\mathcal{H}_{\mathrm{DC}}$ and $\mathcal{H}_{\mathrm{SP}}$,

$$
\dot{P}=-\operatorname{Ln}\left(\partial \mathcal{H}_{\mathrm{DC}}\right)(P), \quad \dot{P}=\operatorname{Ln}\left(\partial \mathcal{H}_{\mathrm{SP}}\right)(P) .
$$

Alternatively, we may write for each $i \in\{1, \ldots, n\}$,

$$
\begin{aligned}
\dot{p}_{i} & =-\pi_{i}\left(\operatorname{Ln}\left(\partial \mathcal{H}_{\mathrm{DC}}\right)\left(p_{1}, \ldots, p_{n}\right)\right), \\
\dot{p}_{i} & =\pi_{i}\left(\operatorname{Ln}\left(\partial \mathcal{H}_{\mathrm{SP}}\right)\left(p_{1}, \ldots, p_{n}\right)\right) .
\end{aligned}
$$

As noted in Section 2.4 these vector fields are discontinuous, and therefore their solution must be understood in the Filippov sense. Eq. (4.7) and Propositions 4.3 and 4.4 provide an expression of the generalized gradients at $P, \partial \mathcal{H}_{\mathrm{DC}}(P)$ and $\partial \mathcal{H}_{\mathrm{SP}}(P)$. One needs to first compute the generalized gradient, then compute the least-norm element, and finally project to each of the $n$ components; therefore the expressions in Proposition 4.6 are not helpful. Note that the least-norm element of convex sets can be computed efficiently, see [6], however closed-form expressions are not available in general.

One can see that the compact set $Q^{n}$ is strongly invariant for both vector fields - $\operatorname{Ln}\left(\partial \mathcal{H}_{\mathrm{DC}}\right)$ and $\operatorname{Ln}\left(\partial \mathcal{H}_{\mathrm{SP}}\right)$. Regarding $-\operatorname{Ln}\left(\partial \mathcal{H}_{\mathrm{DC}}\right)$, this is a consequence of Proposition 4.3 and of Lemma 4.5 Regarding $\operatorname{Ln}\left(\partial \mathcal{H}_{\mathrm{SP}}\right)$, this is a consequence of Proposition 4.4

Proposition 5.1. For the dynamical system (5.2) (respectively (5.3)), the generators' location $P=\left(p_{1}, \ldots, p_{n}\right)$ converges asymptotically to the set of critical points of $\mathcal{H}_{D C}$ (respectively, of $\mathcal{H}_{S P}$ ).

Proof. From Propositions 4.1 and 4.2 we know that $\mathcal{H}_{\mathrm{DC}}$ and $\mathcal{H}_{\mathrm{SP}}$ are globally Lipschitz and regular over $Q^{n}$. The result follows from Proposition 2.11 considering the dynamical system restricted to the strongly invariant and compact domain $Q^{n}$. 口

REMARK 5.2. The gradient dynamical systems enjoy convergence guarantees, but their implementation is centralized because of two reasons. First, all functions $G_{i}(P)$ (respectively $F_{i}(P)$ ) need to be compared in order to determine which generator is active. Second, the least-norm element of the generalized gradients depends on the relative position of the active generators with respect to each other and to the environment.

REMARK 5.3. As illustrated in Fig. 5.1 the evolution of the gradient dynamical systems may not leave fixed even the generators that are centers (circumcenter or incenters). 

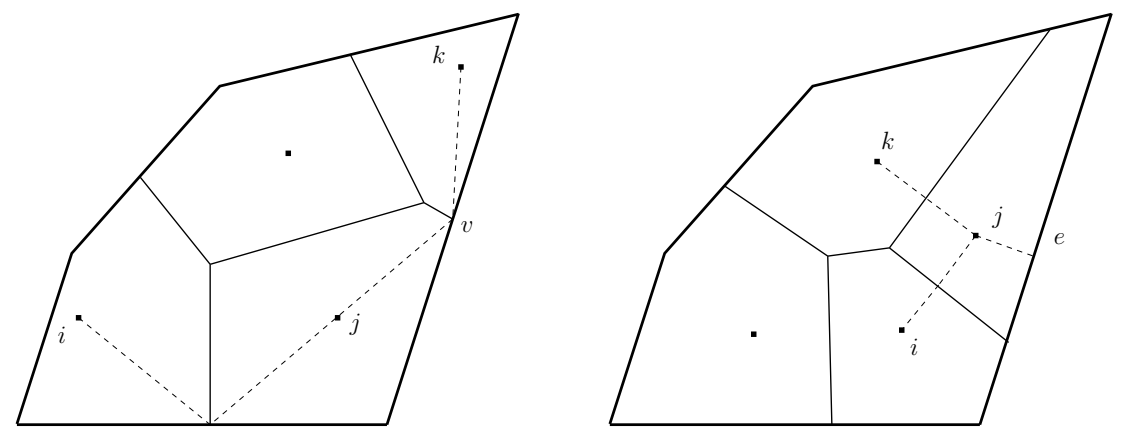

FIG. 5.1. Illustration of the gradient descent. In the left figure, the jth generator is in the circumcenter of its own Voronoi region, but the control law 5.2 drives it toward the vertex $v$. In the right figure, the $j$ th generator is in the incenter of its own Voronoi region, but the control law 5.3 drives it away from the edge e.

5.2. Nonsmooth dynamical systems based on distributed gradients. In this section, we propose a distributed implementation of the previous gradient dynamical systems and explore its relation with behavior-based rules in multiple-vehicle coordination. Consider the following modifications of the gradient dynamical systems (5.2)-(5.3),

$$
\begin{aligned}
& \dot{p}_{i}=-\operatorname{Ln}\left(\partial \lg _{V_{i}(P)}\right)(P), \\
& \dot{p}_{i}=\operatorname{Ln}\left(\partial \operatorname{sm}_{V_{i}(P)}\right)(P),
\end{aligned}
$$

for $i \in\{1, \ldots, n\}$. Note that the system (5.4) is Voronoi-distributed, $\operatorname{since} \operatorname{Ln}\left(\partial \lg _{V_{i}(P)}\right)(P)$ is determined only by the position of $p_{i}$ and of its Voronoi neighbors $\mathcal{N}(P, i)$. On the other hand, the system (5.5) is nearest-neighbor-distributed, since $\operatorname{Ln}\left(\partial \operatorname{sm}_{V_{i}(P)}\right)(P)$ is determined only by the position of $p_{i}$ and its nearest neighbors.

For future reference, let $\operatorname{Ln}\left(\partial \lg _{\mathcal{V}}\right)(P)=\left(\operatorname{Ln}\left(\partial \lg _{V_{1}(P)}\right)(P), \ldots, \operatorname{Ln}\left(\partial \lg _{V_{n}(P)}\right)(P)\right)$, $\operatorname{Ln}\left(\partial \operatorname{sm}_{\mathcal{V}}\right)(P)=\left(\operatorname{Ln}\left(\partial \operatorname{sm}_{V_{1}(P)}\right)(P), \ldots, \operatorname{Ln}\left(\partial \operatorname{sm}_{V_{n}(P)}\right)(P)\right)$, and write

$$
\dot{P}=-\operatorname{Ln}\left(\partial \lg _{\mathcal{V}}\right)(P), \quad \dot{P}=\operatorname{Ln}\left(\partial \operatorname{sm}_{\mathcal{V}}\right)(P) .
$$

As for the previous dynamical systems, note that these vector fields are discontinuous, and therefore their solutions must be understood in the Filippov sense. One can see that the compact set $Q^{n}$ is strongly invariant for both vector fields $-\operatorname{Ln}(\partial \lg \mathcal{V})$ and $\operatorname{Ln}\left(\partial \mathrm{sm}_{\mathcal{V}}\right)$. This fact is a consequence of the expressions for the generalized gradients of $\mathrm{lg}$ and $\mathrm{sm}$ in Proposition 3.3 Note that in the 1-center case, (5.2) (respectively (5.3) ) coincides with (5.4) (respectively with (5.5)).

Proposition 5.4. Let $P \in Q^{n}$. Then the solutions of the dynamical systems (5.4) and (5.5) starting at $P$ are unique.

Proof. (a) Uniqueness of solution for (5.4). Let $D_{\lg }$ be the set of $P \in Q^{n}$ such that $P$ is nondegenerate and $\lg _{V_{i}(P)}\left(p_{i}\right)$ is attained at a single vertex for all $i$. Note that $Q^{n} \backslash D_{\lg }$ has measure zero, and that the vector field $-\operatorname{Ln}\left(\partial \lg _{\mathcal{V}}\right)$ is differentiable (and hence locally Lipschitz) when restricted to any connected component of $D_{\lg }$. Let $P$, $P^{\prime}$ belong to different connected components of $D_{\lg }$, and let $\left\|P-P^{\prime}\right\| \leq \epsilon$. Consider all the indexes $i$ at which the values of $\lg _{V_{i}(P)}\left(p_{i}\right)$ and $\lg _{V_{i}\left(P^{\prime}\right)}\left(p_{i}^{\prime}\right)$ are attained at different vertexes. For these indexes,

$$
-\operatorname{Ln}\left(\partial \lg _{V_{i}(P)}\right)\left(p_{i}\right)+\operatorname{Ln}\left(\partial \lg _{V_{i}\left(P^{\prime}\right)}\right)\left(p_{i}^{\prime}\right)=\operatorname{vrs}\left(v-p_{i}\right)-\operatorname{vrs}\left(w^{\prime}-p_{i}^{\prime}\right),
$$


for certain vertexes $v$ and $w^{\prime}$. Note that for $\epsilon$ small enough, the vertex $w^{\prime}$ in the Voronoi configuration $P^{\prime}$ corresponds to a vertex $w$ in the Voronoi configuration $P$. By construction, $p_{i}$ and $p_{i}^{\prime}$ belong to an $O(\epsilon)$ neighborhood of the bisector $b_{v w}$ determined by $v$ and $w$, and $n_{v w} \cdot\left(p_{i}-p_{i}^{\prime}\right)<0$. In addition, the component of $\operatorname{vrs}\left(v-p_{i}\right)-$ $\operatorname{vrs}\left(w^{\prime}-p_{i}^{\prime}\right)$ along $b_{v w}$ is $O(\epsilon)$ whereas $n_{v w} \cdot \operatorname{vrs}\left(v-p_{i}\right)>0$ and $n_{v w} \cdot \operatorname{vrs}\left(w^{\prime}-p_{i}^{\prime}\right)=$ $n_{v w} \cdot \operatorname{vrs}\left(w-p_{i}\right)+O(\epsilon)$, with $n_{v w} \cdot \operatorname{vrs}\left(w-p_{i}\right)<0$. Then,

$$
\begin{aligned}
& \operatorname{vrs}\left(v-p_{i}\right)-\operatorname{vrs}\left(w^{\prime}-p_{i}^{\prime}\right) \\
& \quad=\operatorname{proj}_{n_{v w}}\left(\operatorname{vrs}\left(v-p_{i}\right)-\operatorname{vrs}\left(w^{\prime}-p_{i}^{\prime}\right)+\operatorname{proj}_{b_{v w}}\left(\operatorname{vrs}\left(v-p_{i}\right)-\operatorname{vrs}\left(w^{\prime}-p_{i}^{\prime}\right)\right.\right. \\
& \quad=\operatorname{proj}_{n_{v w}}\left(\operatorname{vrs}\left(v-p_{i}\right)-\operatorname{vrs}\left(w^{\prime}-p_{i}^{\prime}\right)+O(\epsilon),\right.
\end{aligned}
$$

and, in turn, for sufficiently small $\epsilon$

$$
\begin{aligned}
& \left(p_{i}-p_{i}^{\prime}\right) \cdot\left(\operatorname{vrs}\left(v-p_{i}\right)-\operatorname{vrs}\left(w^{\prime}-p_{i}^{\prime}\right)\right) \\
& \quad=\left(n_{v w} \cdot\left(p_{i}-p_{i}^{\prime}\right)\right)\left(n_{v w} \cdot\left(\operatorname{vrs}\left(v-p_{i}\right)-\operatorname{vrs}\left(w^{\prime}-p_{i}^{\prime}\right)\right)\right)+O\left(\epsilon^{2}\right)<0 .
\end{aligned}
$$

The result now follows from Theorem 1 at page 106 in [15].

(b) Uniqueness of solution for [5.5. Let $D_{\mathrm{sm}}$ be the set of $P \in Q^{n}$ such that $\operatorname{sm}_{V_{i}(P)}\left(p_{i}\right)$ is attained at a single edge for all $i$. Note that $Q^{n} \backslash D_{\mathrm{sm}}$ has measure zero, and that the vector field $\operatorname{Ln}\left(\partial \mathrm{sm}_{\mathcal{V}}\right)$ is differentiable(and hence locally Lipschitz) when restricted to any connected component of $D_{\text {sm }}$. Let $P, P^{\prime}$ belong to different connected components of $D_{\mathrm{sm}}$, and let $\left\|P-P^{\prime}\right\| \leq \epsilon$. Consider all the indexes $i$ at which the values of $\operatorname{sm}_{V_{i}(P)}\left(p_{i}\right)$ and $\operatorname{sm}_{V_{i}\left(P^{\prime}\right)}\left(p_{i}^{\prime}\right)$ are attained at different edges. Assume these edges are of type (a) (the type (b) case can be treated analogously). For these indexes,

$$
\operatorname{Ln}\left(\partial \operatorname{sm}_{V_{i}(P)}\right)\left(p_{i}\right)-\operatorname{Ln}\left(\partial \operatorname{sm}_{V_{i}\left(P^{\prime}\right)}\right)\left(p_{i}^{\prime}\right)=\operatorname{vrs}\left(p_{i}-p_{j}\right)-\operatorname{vrs}\left(p_{i}^{\prime}-p_{k}^{\prime}\right),
$$

for some uniquely determined $p_{j}$ and $p_{k}^{\prime}$, with $j \neq k$. By construction, $p_{i}$ and $p_{i}^{\prime}$ belong to an $O(\epsilon)$ neighborhood of the bisector $b_{j k}$ determined by $p_{j}$ and $p_{k}$, and $n_{k j} \cdot\left(p_{i}-p_{i}^{\prime}\right)<0$. In addition, the component of $\operatorname{vrs}\left(p_{i}-p_{j}\right)-\operatorname{vrs}\left(p_{i}^{\prime}-p_{k}^{\prime}\right)$ along $b_{j k}$ is $O(\epsilon)$ whereas $n_{k j} \cdot \operatorname{vrs}\left(p_{i}-p_{j}\right)>0$ and $n_{k j} \cdot \operatorname{vrs}\left(p_{i}^{\prime}-p_{k}^{\prime}\right)=n_{k j} \cdot \operatorname{vrs}\left(p_{i}-p_{k}\right)+O(\epsilon)$, with $n_{k j} \cdot \operatorname{vrs}\left(p_{i}-p_{k}\right)<0$. Then,

$$
\begin{aligned}
& \operatorname{vrs}\left(p_{i}-p_{j}\right)-\operatorname{vrs}\left(p_{i}^{\prime}-p_{k}^{\prime}\right) \\
& \quad \begin{aligned}
=\operatorname{proj}_{n_{k j}}\left(\operatorname{vrs}\left(p_{i}-p_{j}\right)-\operatorname{vrs}\left(p_{i}^{\prime}\right.\right. & \left.-p_{k}^{\prime}\right)+\operatorname{proj}_{b_{j k}}\left(\operatorname{vrs}\left(p_{i}-p_{j}\right)-\operatorname{vrs}\left(p_{i}^{\prime}-p_{k}^{\prime}\right)\right. \\
& =\operatorname{proj}_{n_{k j}}\left(\operatorname{vrs}\left(p_{i}-p_{j}\right)-\operatorname{vrs}\left(p_{i}-p_{k}\right)+O(\epsilon),\right.
\end{aligned}
\end{aligned}
$$

and, in turn, for sufficiently small $\epsilon$

$$
\begin{aligned}
& \left(p_{i}-p_{i}^{\prime}\right) \cdot\left(\operatorname{vrs}\left(p_{i}-p_{j}\right)-\operatorname{vrs}\left(p_{i}^{\prime}-p_{k}^{\prime}\right)\right) \\
& \quad=\left(n_{k j} \cdot\left(p_{i}-p_{i}^{\prime}\right)\right)\left(n_{k j} \cdot\left(\operatorname{vrs}\left(p_{i}-p_{j}\right)-\operatorname{vrs}\left(p_{i}-p_{k}\right)\right)\right)+O\left(\epsilon^{2}\right)<0 .
\end{aligned}
$$

The result now follows from Theorem 1 at page 106 in [15].

REMARK 5.5 (Relation with behavior-based robotics: move toward the furthestaway vertex). The distributed gradient control law in the disk-covering setting (5.4) has an interesting interpretation in the context of behavior-based robotics. Consider the $i$ th generator. If the maximum of $\lg _{V_{i}(P)}$ is attained at a single vertex $v$ of its Voronoi cell $V_{i}$, then $\lg _{V_{i}(P)}$ is differentiable at that configuration, and its derivative 
corresponds to $\operatorname{vrs}\left(p_{i}-v\right)$. Therefore, the control law (5.4) corresponds to the behavior "move toward the furthest vertex in own Voronoi cell." If there are two or more vertexes of $V_{i}$ where the value $\lg _{V_{i}(P)}\left(p_{i}\right)$ is attained, then (15.4) provides an average behavior by computing the least-norm element in the convex hull of all $\operatorname{vrs}\left(p_{i}-v\right)$ such that $\left\|p_{i}-v\right\|=\lg _{V_{i}(P)}\left(p_{i}\right)$.

REMARK 5.6 (Relation with behavior-based robotics: move away from the nearest neighbor). The distributed gradient control law in the sphere-packing setting (5.5) has also an interesting interpretation. For the $i$ th generator, if the minimum of $\operatorname{sm}_{V_{i}(P)}$ is attained at a single edge $e$, then $\operatorname{sm}_{V_{i}(P)}$ is differentiable at that configuration, and its derivative is $n_{e}$. The control law (5.5) corresponds to the behavior "move away from the nearest neighbor" (where a neighbor can also be the boundary of the environment). If there are two or more edges where the value $\operatorname{sm}_{V_{i}(P)}\left(p_{i}\right)$ is attained, then (5.5) provides an average behavior in an analogous manner as before.

Proposition 5.7. For the dynamical system (5.4), the generators' location $P=$ $\left(p_{1}, \ldots, p_{n}\right)$ converges asymptotically to the largest weakly invariant set contained in the closure of $A_{\mathrm{DC}}(Q)=\left\{P \in Q^{n} \mid i \in I(P) \Longrightarrow p_{i}=\mathrm{CC}\left(V_{i}\right)\right\}$.

Proof. Let $a \in \widetilde{\mathcal{L}}_{-\operatorname{Ln}\left(\partial \lg _{\mathcal{V}}\right)} \mathcal{H}_{\mathrm{DC}}(P)$. By definition, $a=-\operatorname{Ln}\left(\partial \lg _{\mathcal{V}}\right)(P) \cdot \zeta$, for all $\zeta \in \partial \mathcal{H}_{\mathrm{DC}}(P)$. Let $v \in \operatorname{Ve}_{\mathrm{DC}}(\mathcal{V}(P))$. From Lemmas 4.3 and 4.5 we know that, independently of the degenerate/nondegenerate character of the Voronoi partition at $v$, there always exist either an edge $e$ of $Q$ and generators $p_{i}$ and $p_{j}$, or generators $p_{i}, p_{j}$ and $p_{k}$, such that $\lambda(e, i, j), \lambda(e, j, i)>0$ (respectively $\mu(i, j, k), \mu(j, k, i), \mu(k, i, j)>$ $0)$. If $v$ is a vertex of type (b), then

$$
\begin{aligned}
a & =-\operatorname{Ln}\left(\partial \lg _{\mathcal{V}}\right)(P) \cdot \partial_{v} G_{i} \\
& =-\operatorname{Ln}\left(\partial \lg _{V_{i}(P)}\right)(P) \cdot \lambda(e, i, j) \operatorname{vrs}\left(p_{i}-v\right)-\operatorname{Ln}\left(\partial \lg _{V_{j}(P)}\right)(P) \cdot \lambda(e, j, i) \operatorname{vrs}\left(p_{j}-v\right)
\end{aligned}
$$

From Lemma 3.8(i) we conclude that $a \leq 0$, and the inequality is strict if either $p_{i} \neq \mathrm{CC}\left(V_{i}\right)$ or $p_{j} \neq \mathrm{CC}\left(V_{j}\right)$. The same conclusion can be derived if $v$ is a vertex of type (a). Therefore, $\max \widetilde{\mathcal{L}}_{-\operatorname{Ln}(\partial \lg \mathcal{V})} \mathcal{H}_{\mathrm{DC}}(P) \leq 0$ or $\widetilde{\mathcal{L}}_{-\operatorname{Ln}(\partial \lg \mathcal{V})} \mathcal{H}_{\mathrm{DC}}(P)=\emptyset$. Now, resorting to the LaSalle principle (Theorem 2.9), we deduce that the solution $P:[0,+\infty) \rightarrow Q^{n}$ starting from $P_{0}$ converges to the largest weakly invariant set

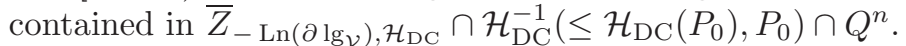

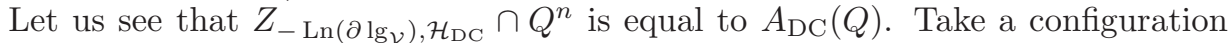
$P \in A_{\mathrm{DC}}(Q)$. Then, $\operatorname{Ln}\left(\partial \lg _{V_{i}(P)}\right)(P)=0$ if $i \in I(P)$, and $\pi_{i}(\zeta)=0$ if $i \notin I(P)$, for any $\zeta \in \partial \mathcal{H}_{\mathrm{DC}}(P)$ (cf. Proposition 4.6). Consequently, $0=-\operatorname{Ln}(\partial \lg \mathcal{V})(P) \cdot \zeta$, for all $\zeta \in \partial \mathcal{H}_{\mathrm{DC}}(P)$, and so $0 \in \widetilde{\mathcal{L}}_{-\operatorname{Ln}\left(\partial \lg _{\mathcal{V}}\right)} \mathcal{H}_{\mathrm{DC}}(P)$. Therefore, $A_{\mathrm{DC}}(Q) \subset Z_{-\operatorname{Ln}(\partial \lg \mathcal{V}), \mathcal{H}_{\mathrm{DC}}}$. Now, consider $P \in Z_{-\operatorname{Ln}\left(\partial \lg _{\mathcal{V}}\right), \mathcal{H}_{\mathrm{DC}}}$. Then, $0 \in \widetilde{\mathcal{L}}_{-\operatorname{Ln}(\partial \lg \mathcal{V})} \mathcal{H}_{\mathrm{DC}}(P)$, that is, $0=$ $-\operatorname{Ln}\left(\partial \lg _{\mathcal{V}}\right)(P) \cdot \zeta$, for all $\zeta \in \partial \mathcal{H}_{\mathrm{DC}}(P)$. If $P$ is nondegenerate, we deduce from eq. (5.6) and Lemma 3.8 that all the active generators are centered, i.e., $P \in A_{\mathrm{DC}}(Q)$. If $P$ is degenerate, consider a degenerate vertex $v$ where the value of $\mathcal{H}_{\mathrm{DC}}(P)$ is attained. For simplicity, we deal with the case where $v$ is contained in an edge $e$ of $Q$ (the case $v \in \operatorname{int}(Q)$ is treated analogously). From Lemma 4.5 we know that there exist generators $p_{i}, p_{j}$ determining $v$ on opposite sides of $l$, the orthogonal line to the edge $e$ passing through $v$. From eq. (5.6) and Lemma 3.8 we deduce that both $p_{i}$ and $p_{j}$ are centered. Now, for each generator $p_{k}$ with $v \in V_{k}$ in the same side of $l$ as $p_{i}$ (respectively $p_{j}$ ), we consider the triplet $(e, j, k)$ (respectively $(e, i, k)$ ). Again resorting to eq. (5.6) and Lemma 3.8 we conclude that $p_{k}$ is also centered. Finally, if a generator $p_{k}$ with $v \in V_{k}$ is such that $p_{k} \in l$, any of the triplets $(e, j, k)$ or $(e, i, k)$ can be invoked in a similar argument to ensure that $p_{k}$ is centered. Therefore, 


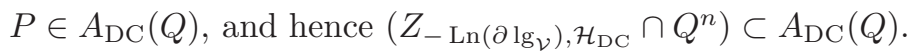

Proposition 5.8. For the dynamical system [5.5), the generators' location $P=$ $\left(p_{1}, \ldots, p_{n}\right)$ converges asymptotically to the largest weakly invariant set contained in the closure of $A_{\mathrm{SP}}(Q)=\left\{P \in Q^{n} \mid i \in I(P) \Longrightarrow p_{i} \in \operatorname{IC}\left(V_{i}\right)\right\}$.

Proof. Let $a \in \widetilde{\mathcal{L}}_{\operatorname{Ln}\left(\partial \mathrm{sm}_{\mathcal{V}}\right)} \mathcal{H}_{\mathrm{SP}}(P)$. By definition, $a=\operatorname{Ln}\left[\operatorname{sm}_{\mathcal{V}}\right](P) \cdot \zeta$, for all $\zeta \in \partial \mathcal{H}_{\mathrm{SP}}(P)$. Let $e \in \operatorname{Ed}_{\mathrm{SP}}(\mathcal{V}(P))$. If $e$ is an edge of type (a), i.e. a segment of the bisector determined by $p_{i}$ and $p_{j}$, we compute (cf. Proposition 4.4),

$$
\begin{aligned}
a & =\operatorname{Ln}\left(\partial \operatorname{sm}_{\mathcal{V}}\right)(P) \cdot \partial_{e} F_{i} \\
& =\operatorname{Ln}\left(\partial \operatorname{sm}_{V_{i}(P)}\right)(P) \cdot \pi_{i}\left(\partial_{e} F_{i}\right)+\operatorname{Ln}\left(\partial \operatorname{sm}_{V_{j}(P)}\right)(P) \cdot \pi_{j}\left(\partial_{e} F_{i}\right) .
\end{aligned}
$$

From Lemma 3.8 (iii) we conclude that $a \geq 0$, and the inequality is strict if either $p_{i} \notin \operatorname{IC}\left(V_{i}\right)$ or $p_{j} \notin \operatorname{IC}\left(V_{j}\right)$. The same conclusion can be derived if $e$ is a vertex of type (b). Therefore, $\max \widetilde{\mathcal{L}}_{\operatorname{Ln}\left(\partial \mathrm{sm}_{\mathcal{V}}\right)} \mathcal{H}_{\mathrm{SP}}(P) \geq 0$ or $\widetilde{\mathcal{L}}_{\mathrm{Ln}\left(\partial \mathrm{sm}_{\mathcal{V}}\right)} \mathcal{H}_{\mathrm{SP}}(P)=\emptyset$. Now, resorting to the LaSalle principle (Theorem [2.9), we deduce that the solution $P:[0,+\infty) \rightarrow Q^{n}$ starting from $P_{0}$ converges to the largest weakly invariant set con-

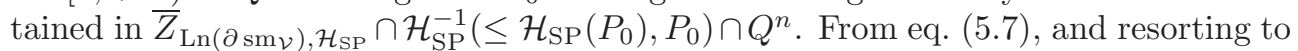
Proposition 4.6] and Lemma 3.8 one can also show that $Z_{\operatorname{Ln}(\partial \operatorname{sm} \nu)}, \mathcal{H}_{\mathrm{SP}} \cap Q^{n}$ is equal to $A_{\mathrm{SP}}(Q)$.

REMARK 5.9. The sets $A_{\mathrm{DC}}(Q)$ and $A_{\mathrm{SP}}(Q)$ are not closed in general. If $\operatorname{dim} Q=$ 1 , then it can be seen that they indeed are. In higher dimensions one can find sequences $\left\{P_{k} \in Q^{n} \mid k \in \mathbb{N}\right\}$ in these sets which converge to configurations $P$ where not all active generators are centered.

5.3. Distributed dynamical systems based on geometric centering. Here, we propose alternative distributed dynamical systems for the multi-center functions. Our design is directly inspired by the results in Theorems 4.7 and 4.8 on the critical points of the multi-center functions $\mathcal{H}_{\mathrm{DC}}$ and $\mathcal{H}_{\mathrm{SP}}$. For $i \in\{1, \ldots, n\}$, consider the dynamical systems

$$
\begin{gathered}
\dot{p}_{i}=\operatorname{CC}\left(V_{i}\right)-p_{i}, \\
\dot{p}_{i} \in \operatorname{IC}\left(V_{i}\right)-p_{i} .
\end{gathered}
$$

Alternatively, we may write $\dot{P}=\mathrm{CC}(\mathcal{V}(P))-P$ and $\dot{P} \in \operatorname{IC}(\mathcal{V}(P))-P$. Note that both systems are Voronoi-distributed. Also, note that the vector field [5.8) is continuous, since the circumcenter of a polygon depends continuously on the location of its vertexes, and the location of the vertexes of the Voronoi partition depends continuously on the location of the generators; see 23]. However, eq. (5.9) is a differential inclusion, since the incenter sets may not be singletons. By Lemma 2.7 the existence of solutions to eq. (5.9) is guaranteed by the following result.

Proposition 5.10. Consider the set-valued map $\operatorname{IC}(\mathcal{V})-\operatorname{Id}: Q^{n} \rightarrow 2^{\left(\mathbb{R}^{2}\right)^{n}}$ given by $P \mapsto \operatorname{IC}(\mathcal{V}(P))-P$. Then $\operatorname{IC}(\mathcal{V})-\mathrm{Id}$ is upper semicontinuous with nonempty, compact and convex values.

Proof. Clearly, the map $\operatorname{IC}(\mathcal{V})-\operatorname{Id}$ takes nonempty and compact values. From Lemma 3.2 we also know that it takes convex values. Furthermore, since the identity map is continuous, it suffices to check that $P \mapsto \operatorname{IC}(\mathcal{V}(P))$ is upper semicontinuous. We then have to verify that, given $P_{0} \in Q^{n}$, for each $\epsilon>0$, there exists $\delta>0$ such that

$$
\mathrm{IC}(\mathcal{V}(P)) \subset \operatorname{IC}\left(\mathcal{V}\left(P_{0}\right)\right)+B_{2 n}(0, \epsilon), \quad \text { if }\left\|P-P_{0}\right\| \leq \delta .
$$


Now, for each $i$, if $\operatorname{IC}\left(V_{i}\left(P_{0}\right)\right)$ is not a singleton, then it is a segment (cf. Lemma 3.2) whose extremal points $q_{i 1}\left(P_{0}\right), q_{i 2}\left(P_{0}\right)$ are the intersection points of some bisectors of the edges of the Voronoi cell. It is clear that $q_{i \alpha}(P) \rightarrow q_{i \alpha}\left(P_{0}\right)$ when $P \rightarrow P_{0}$ for $\alpha=1,2$. Therefore, given $\epsilon>0$, one can choose $\delta_{i}>0$ such that if $\left\|P-P_{0}\right\| \leq$ $\delta_{i}$, then $\left\|q_{i \alpha}(P)-q_{i \alpha}\left(P_{0}\right)\right\| \leq \epsilon / n$. Since $\operatorname{IC}\left(V_{i}(P)\right)$ is contained in the segment joining $q_{i 1}(P)$ and $q_{i 2}(P)$, we deduce $\operatorname{IC}\left(V_{i}(P)\right) \subset \operatorname{IC}\left(V_{i}\left(P_{0}\right)\right)+B_{2}(0, \epsilon / n)$. On the other hand, if $\operatorname{IC}\left(V_{i}\left(P_{0}\right)\right)$ is a singleton, then it coincides with the intersection points $q_{i 1}\left(P_{0}\right), \ldots, q_{i m}\left(P_{0}\right)$ of some bisectors of the edges of the Voronoi cell. The above reasoning also guarantees that there exits $\delta_{i}>0$ such that $q_{i \alpha}(P) \in \operatorname{IC}\left(V_{i}\left(P_{0}\right)\right)+$ $B_{2}(0, \epsilon / n), \alpha=1, \ldots, m$, if $\left\|P-P_{0}\right\| \leq \delta_{i}$. Since $\operatorname{IC}\left(V_{i}(P)\right)$ is contained in one of the segments joining the points $q_{i 1}(P), \ldots, q_{i m}(P)$, we again deduce $\operatorname{IC}\left(V_{i}(P)\right) \subset$ $\mathrm{IC}\left(V_{i}\left(P_{0}\right)\right)+B_{2}(0, \epsilon / n)$. The statement in f5.10 follows by taking the minimum of $\delta_{1}, \ldots, \delta_{n}$.

Having established the existence of solutions, one can also see that the compact set $Q^{n}$ is strongly invariant for the vector field $\operatorname{CC}(\mathcal{V})$ - Id and for the differential inclusion $\operatorname{IC}(\mathcal{V})-$ Id. Next, we characterize the asymptotic convergence of the dynamical systems under study.

Proposition 5.11. For the dynamical system (5.8) (respectively (5.9)), the generators' location $P=\left(p_{1}, \ldots, p_{n}\right)$ converges asymptotically to the largest weakly invariant set contained in the closure of $A_{\mathrm{DC}}(Q)$ (respectively in the closure of $A_{\mathrm{SP}}(Q)$ ).

Proof. The proof of this result is parallel to the proofs of Propositions 5.7 and 5.8 The sequence of steps is the same as before, though now one resorts to Lemma 3.8 (ii) and Lemma 3.8(iv). The only additional observation is that, when computing the set-valued Lie derivative for eq. (5.9), one has that $a \in \widetilde{\mathcal{L}}_{\mathrm{IC}(\mathcal{V})-\mathrm{Id}} \mathcal{H}_{\mathrm{SP}}(P)$ if and only if there exists $x \in \operatorname{IC}(\mathcal{V}(P))$ such that $a=(x-P) \cdot \zeta$, for any $\zeta \in \partial \mathcal{H}_{\mathrm{SP}}(P)$. The application of Lemma 3.8 guarantees that $a \geq 0$, and that the inequality is strict if any of the active generators is not in its corresponding incenter set.

5.4. Simulations. To illustrate the performance of the distributed coordination algorithms, we include some simulation results. The algorithms are implemented in Mathematica as a single centralized program. We compute the bounded Voronoi diagram of a collection of points using the Mathematica package ComputationalGeometry. We compute the circumcenter of a polygon via the algorithm in [28] and the incenter set via the LinearProgramming solver in Mathematica. Measuring displacements in meters, we consider the domain determined by the vertexes

$$
\{(0,0),(2.5,0),(3.45,1.5),(3.5,1.6),(3.45,1.7),(2.7,2.1),(1 ., 2.4),(.2,1.2)\} .
$$

In Figs. 5.2 and 5.3 we illustrate the performance of the dynamical systems (5.4) and (5.8), respectively, minimizing the multi-circumcenter function $\mathcal{H}_{\text {DC. }}$ In Figs. [5.4 and [5.5 we illustrate the performance of the dynamical systems (5.5) and (5.9), respectively, maximizing the multi-incenter function $\mathcal{H}_{\mathrm{SP}}$. Observing the final configurations in the four figures, one can verify, visually and numerically, that the active generators are asymptotically centered as forecast by our analysis.

6. Conclusions. We have introduced two multi-center functions that provide quality-of-service measures for mobile networks. We have shown that both functions are globally Lipschitz and regular, and we have computed their generalized gradients. Furthermore, under certain technical conditions, we have characterized via nonsmooth analysis their critical points as center Voronoi configurations and as solutions of diskcovering and sphere-packing problems. We have also considered various algorithms 

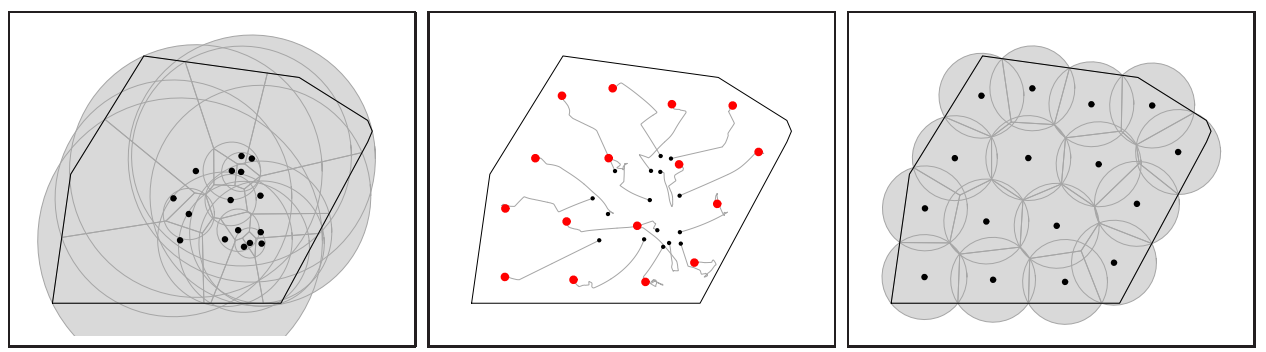

FIG. 5.2. "Toward the furthest" algorithm for 16 generators in a convex polygonal environment. The left (respectively, right) figure illustrates the initial (respectively, final) locations and Voronoi partition. The central figure illustrates the network evolution. After 2 seconds, the multi-center function is approximately 39504 meters.
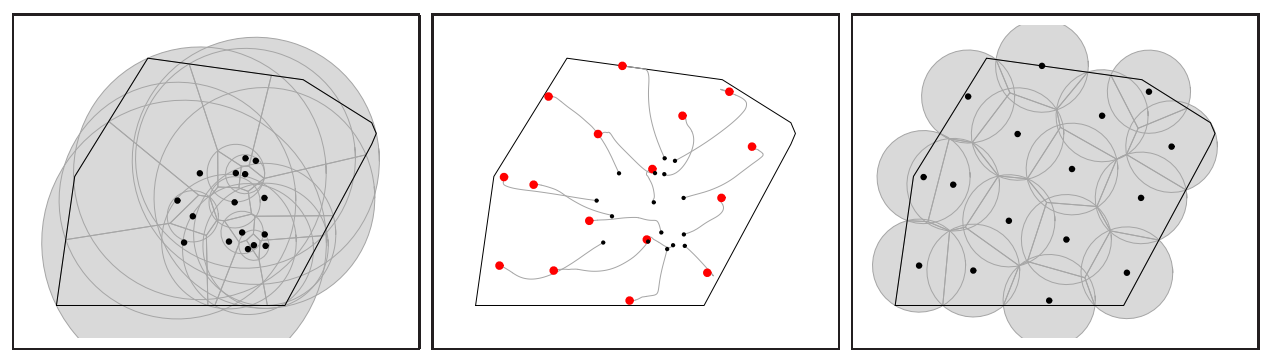

FIG. 5.3. "Move-toward-the-circumcenter" algorithm for 16 generators in a convex polygonal environment. The left (respectively, right) figure illustrates the initial (respectively, final) locations and Voronoi partition. The central figure illustrates the network evolution. After 20 seconds, the multi-center function is approximately 0.43273 meters.

that extremize the multi-center functions. First, we considered the nonsmooth gradient flows induced by their respective generalized gradients. Second, we devised a novel strategy based on the generalized gradients of the 1-center functions of each generator. Third, we introduced and characterized a geometric centering strategy with resemblances to the classical Lloyd algorithm. We have unveiled the remarkable geometric interpretations of these algorithms, discussed their distributed character and analyzed their asymptotic behavior using nonsmooth stability analysis.

Future directions of research include: (i) sharpening the asymptotic convergence results for the proposed dynamical systems, (ii) considering the setting of convex polytopes in $\mathbb{R}^{N}$, for $N>2$, (iii) understanding in what sense the proposed multicircumcenter and the multi-incenter problems can be shown to be dual, and (iv) analyzing other meaningful geometric optimization problems and their relations with cooperative behaviors.

\section{REFERENCES}

[1] P. K. Agarwal And M. Sharir, Efficient algorithms for geometric optimization, ACM Computing Surveys, 30 (1998), pp. 412-458.

[2] R. C. Arkin, Behavior-Based Robotics, Cambridge University Press, New York, NY, 1998.

[3] A. Bacciotti and F. Ceragioli, Stability and stabilization of discontinuous systems and nonsmooth Lyapunov functions, ESAIM. Control, Optimisation \& Calculus of Variations, 4 (1999), pp. 361-376.

[4] D. P. Bertsekas and J. N. Tsitsiklis, Parallel and Distributed Computation: Numerical Methods, Athena Scientific, 1997. 

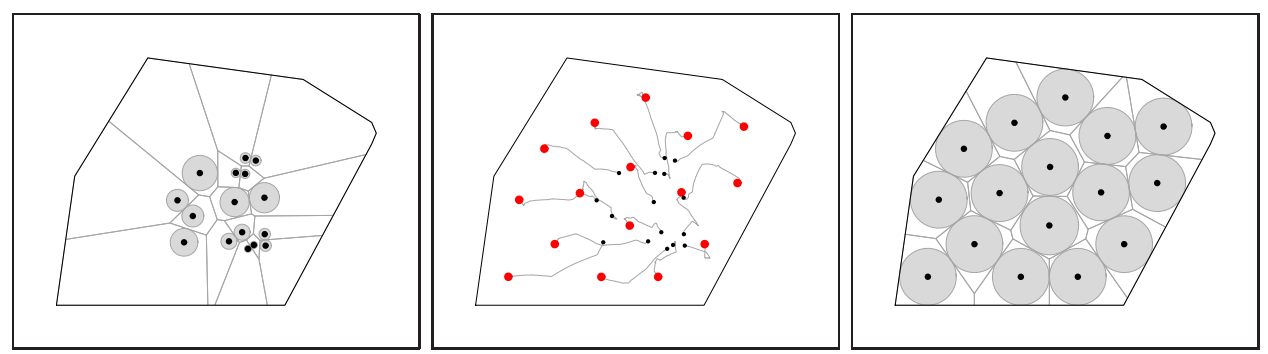

FIG. 5.4. "Away-from-closest" algorithm for 16 generators in a convex polygonal environment. The left (respectively, right) figure illustrates the initial (respectively, final) locations and Voronoi partition. The central figure illustrates the network evolution. After 2 seconds, the multi-center function is approximately 26347 meters.
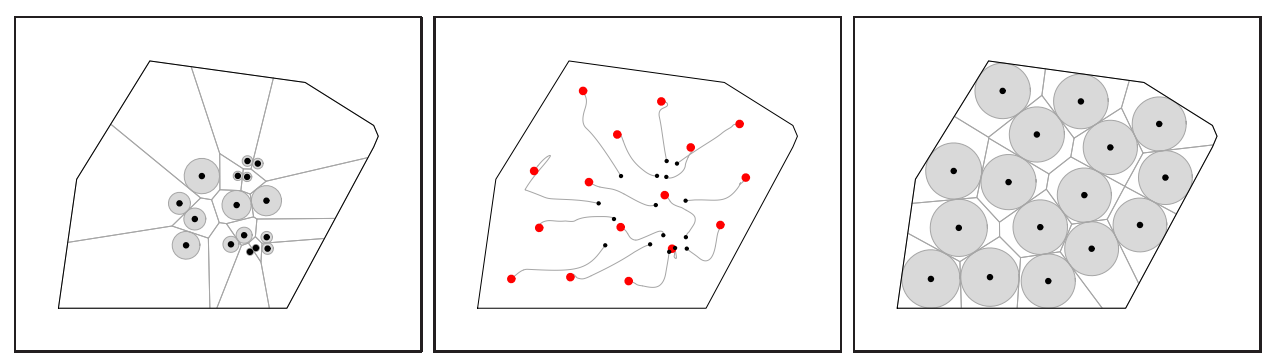

FIG. 5.5. "Move-toward-the-incenter" algorithm for 16 generators in a convex polygonal environment. The left (respectively, right) figure illustrates the initial (respectively, final) locations and Voronoi partition. The central figure illustrates the network evolution. After 20 seconds, the multi-center function is approximately 2498 meters.

[5] V. Boltyanski, H. Martini, and V. Soltan, Geometric methods and optimization problems, vol. 4 of Combinatorial optimization, Kluwer Academic Publishers, Dordrecht, Boston, 1999.

[6] S. Boyd and L. Vandenberghe, Convex optimization. Preprint, Dec. 2002.

[7] R. W. Brockett, Dynamical systems that sort lists, diagonalize matrices, and solve linear programming problems, Linear Algebra and its Applications, 146 (1991), pp. 79-91.

[8] H. CHOSET, Nonsmooth analysis, convex analysis, and their applications to motion planning, International Journal of Computational Geometry and Applications, 9 (1999), pp. 447-469.

[9] F. H. Clarke, Optimization and Nonsmooth Analysis, Canadian Mathematical Society Series of Monographs and Advanced Texts, John Wiley \& Sons, 1983.

[10] J. Cortés, S. Martínez, T. Karatas, and F. Bullo, Coverage control for mobile sensing networks, IEEE Transactions on Robotics and Automation, (2002). Conditionally accepted.

[11] M. de Berg, M. van Kreveld, and M. Overmars, Computational Geometry: Algorithms and Applications, Springer Verlag, New York, NY, 1997.

[12] J. P. Desai, J. P. Ostrowski, And V. Kumar, Modeling and control of formations of nonholonomic mobile robots, IEEE Transactions on Robotics and Automation, 17 (2001), pp. 905-8.

[13] Z. Drezner, ed., Facility Location: A Survey of Applications and Methods, Springer Series in Operations Research, Springer Verlag, New York, NY, 1995.

[14] Q. Du, V. Faber, and M. Gunzburger, Centroidal Voronoi tessellations: applications and algorithms, SIAM Review, 41 (1999), pp. 637-676.

[15] A. F. Filippov, Differential Equations with Discontinuous Righthand Sides, vol. 18 of Mathematics and Its Applications, Kluwer Academic Publishers, Dordrecht, 1988. Original Russian edition: Differentsial'nye Uravneniya s Razryvnoi Pravoi Chast'yu, Nauka, Moscow, 1985.

[16] R. M. Gray and D. L. Neuhoff, Quantization, IEEE Transactions on Information Theory, 44 (1998), pp. 2325-2383. Commemorative Issue 1948-1998.

[17] U. Helmke And J. Moore, Optimization and Dynamical Systems, Springer Verlag, New York, NY, 1994. 
[18] A. Jadbabaie, J. Lin, And A. S. Morse, Coordination of groups of mobile autonomous agents using nearest neighbor rules, IEEE Transactions on Automatic Control, (2003). To appear.

[19] N. E. LeOnard And E. Fiorelli, Virtual leaders, artificial potentials, and coordinated control of groups, in IEEE Conf. on Decision and Control, Orlando, FL, Dec. 2001, pp. 2968-2973.

[20] Y. Liu, K. M. Passino, And M. M. Polycarpou, Stability analysis of m-dimensional asynchronous swarms with a fixed communication topology, IEEE Transactions on Automatic Control, 48 (2003), pp. 76-95.

[21] S. P. LLOYD, Least squares quantization in PCM, IEEE Transactions on Information Theory, 28 (1982), pp. 129-137. Presented as Bell Laboratory Technical Memorandum at a 1957 Institute for Mathematical Statistics meeting.

[22] D. G. Luenberger, Linear and Nonlinear Programming, Addison-Wesley, Reading, Massachusetts, second ed., 1984.

[23] A. Okabe, B. Boots, K. Sugihara, and S. N. Chiu, Spatial Tessellations: Concepts and Applications of Voronoi Diagrams, Wiley Series in Probability and Statistics, John Wiley \& Sons, New York, NY, second ed., 2000.

[24] R. Olfati-Saber and R. M. Murray, Agreement problems in networks with directed graphs and switching topology, in IEEE Conf. on Decision and Control, 2003. Submitted.

[25] B. PAden AND S. S. SASTRY, A calculus for computing Filippov's differential inclusion with application to the variable structure control of robot manipulators, IEEE Transactions on Circuits and Systems, 34 (1987), pp. 73-82.

[26] J.-M. Robert and G. T. Toussaint, Computational geometry and facility location, in Proc. International Conf. on Operations Research and Management Science, vol. B, Manila, The Philippines, Dec. 1990, pp. 1-19.

[27] D. Shevitz and B. Paden, Lyapunov stability theory of nonsmooth systems, IEEE Transactions on Automatic Control, 39 (1994), pp. 1910-1914.

[28] S. Skyum, A simple algorithm for computing the smallest circle, Information Processing Letters, 37 (1991), pp. 121-125.

[29] A. Suzuki and Z. Drezner, The p-center location problem in an area, Location Science, 4 (1996), pp. 69-82.

[30] I. Suzuki And M. Yamashita, Distributed anonymous mobile robots: Formation of geometric patterns, SIAM Journal on Computing, 28 (1999), pp. 1347-1363.

[31] H. Tanner, A. Jadbabaie, and G. J. Pappas, Stable flocking of mobile agents, Part II: dynamic topology, in IEEE Conf. on Decision and Control, Maui, Hawaii, Dec. 2003. Submitted. 


\section{Symbol Description and page(s) when applicable}

$A_{\mathrm{DC}}(Q)$ Set of configurations $P \in Q^{n}$ where all active generators are in the circumcenter of its own Voronoi region, 25

$A_{\mathrm{SP}}(Q)$ Set of configurations $P \in Q^{n}$ where all active generators are in the incenter set of its own Voronoi region, 26

$\mathrm{CC}(Q)$ Circumcenter of polytope $Q, 6$

$\operatorname{CR}(Q)$ Circumradius of polytope $Q, 6$

$\mathrm{D}_{S}$ Distance function to the convex set $S, 4$

$\operatorname{Ed}(Q)$ Edges of polygon $Q, 4$

$\operatorname{Ed}_{\mathrm{SP}}(\mathcal{V}(P))$ Edges where the value of $\mathcal{H}_{\mathrm{SP}}(P)$ is attained, 6

$e(i)$ Edge of $\mathcal{V}(P)$ belonging to $V_{i}$ and to the boundary of $Q, 5$

$e(i, j)$ Edge of $\mathcal{V}(P)$ determined by $p_{i}$ and $p_{j}, 5$

$F_{i}(P)$ Smallest distance from $p_{i}$ to the boundary of $V_{i}(P), 14$

$G_{i}(P)$ Largest distance from $p_{i}$ to the boundary of $V_{i}(P), 14$

$\partial f$ Generalized gradient of the locally Lipschitz function $f, 7$

$\mathcal{H}_{\text {DC }}$ Multi-circumcenter function, 6

$\mathcal{H}_{\mathrm{SP}}$ Multi-incenter function, 6

$\operatorname{IC}(Q)$ Incenter set of polytope $Q, 6$

$\operatorname{IR}(Q)$ Inradius of polytope $Q, 6$

$K[X]$ Filippov mapping associated with a measurable and essentially locally bounded mapping $X: \mathbb{R}^{N} \rightarrow \mathbb{R}^{N}, 8$

$\lambda(e, i, j)$ Scalar function associated with the vertex $v(e, i, j), 16$

$\operatorname{Ln}(S)$ Least-norm element of the convex set $S, 7$

$\lg _{Q}(p)$ Largest distance from $p$ to the boundary of $Q, 10$

$\mu(i, j, k)$ Scalar function associated with the vertex $v(i, j, k), 17$

$\mathcal{N}(P, i), \mathcal{N}(i)$ Set of neighbors of the $i$ th generator at configuration $P, 4$

$n_{e(i, j)}$ Unit normal to $e(i, j)$ pointing toward $\operatorname{int}\left(V_{i}(P)\right), 5$

$n_{e(i)}$ Unit normal to $e(i)$ pointing toward $\operatorname{int}(Q), 5$

$\operatorname{proj}_{S}$ Orthogonal projection onto the convex set $S, 4$

$\pi_{i}$ Canonical projection from $Q^{n}$ onto the $i$ th factor, 4

$\widetilde{\mathcal{L}}_{X} f$ Set-valued Lie derivative of $f$ with respect to $X, 8$

$\operatorname{sm}_{Q}(p)$ Smallest distance from $p$ to the boundary of $Q, 10$

$v(i, j, k)$ Vertex of $\mathcal{V}(P)$ determined by $p_{i}, p_{j}$ and $p_{k}, 4$

$v(e, i, j)$ Vertex of $\mathcal{V}(P)$ determined by $e \in \operatorname{Ed}(Q)$ and $p_{i}, p_{j}, 4$

$v(e, f, i)$ Vertex of $\mathcal{V}(P)$ determined by $e, f \in \operatorname{Ed}(Q)$ and $p_{i}, 4$

$\operatorname{Ve}_{\mathrm{DC}}(\mathcal{V}(P))$ Vertexes of $\mathcal{V}(P)$ where the value of $\mathcal{H}_{\mathrm{DC}}(P)$ is attained, 6

$\operatorname{vrs}(v)$ Unit vector in the direction of $0 \neq v \in \mathbb{R}^{N}, 4$

$\operatorname{Ve}(Q)$ Vertexes of polygon $Q, 4$

$\mathcal{V}(P)$ Voronoi partition of $Q$ generated by $P=\left(p_{1}, \ldots, p_{n}\right), 4$

$Z_{X, f}$ Set formed by points $x \in \mathbb{R}^{N}$ such that 0 belongs to $\widetilde{\mathcal{L}}_{X} f(x), 9$ 